\title{
Process-based modelling of bank-breaking mechanisms of tidal sandbanks
}

\author{
Thomas J. van Veelen ${ }^{\mathrm{a}, \mathrm{b}}$, Pieter C. Roos ${ }^{\mathrm{a}, *}$, Suzanne J.M.H. Hulscher ${ }^{\mathrm{a}}$

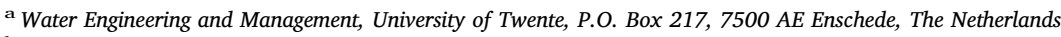 \\ ${ }^{\mathrm{b}}$ Zienkiewicz Centre for Computational Engineering, College of Engineering, Bay Campus, Swansea University, Fabian Way, Swansea SA1 8EN, UK
}

\section{A R T I C L E I N F O}

\section{Keywords:}

Tidal sandbanks

Process-based modelling

Nonlinear morphodynamics

Transient evolution

Bank-breaking mechanisms

S-shape

\begin{abstract}
A B S T R A C T
Tidal sandbanks are large-scale dynamic bed forms observed in shallow shelf seas. Their plan view evolution may display a single bank breaking into two or more banks, for which two mechanisms have been proposed in the literature. However, as both were based on interpretation of observations, generic support from a processbased modelling perspective is lacking so far. Here we present a new idealised process-based model study into the transient evolution of tidal sandbanks. Key elements are the inclusion of nonlinear dynamics for topographies that vary in both horizontal directions, and the focus on long-term evolution (centuries and longer). As a further novelty, the hydrodynamic solution, satisfying the nonlinear shallow water equations including bottom friction and the Coriolis effect, is obtained from a truncated expansion in the ratio of maximum bank elevation (w.r.t. mean depth) and mean water depth. Bed evolution follows from the tidally averaged bed load sediment transport, enhanced by depth-dependent wind-wave stirring. From our model results, we identify two paths of evolution, leading to either bank-breaking or an S-shape. Which of these paths occurs depends on initial topography, with bank orientation and bank length as major control parameters. The breaking and S-shape obtained in our model results show resemblance with banks observed in the North Sea.
\end{abstract}

\section{Introduction}

Tidal sandbanks occur in patches throughout shallow shelf seas, such as the North Sea. With lengths in the order of tens of kilometres, widths of five to ten kilometres and heights of tens of metres, they are the largest in the class of tide-driven rhythmic bottom features (Dyer and Huntley, 1999, who use the term 'open shelf ridges'). Sandbanks in the Northern Hemisphere generally have an orientation of 0-20 degrees anticlockwise with respect to the principal tidal current (Kenyon et al., 1981). Their slow evolution, typically on a time scale of centuries, makes it unclear whether they are in equilibrium (Dyer and Huntley, 1999).

Understanding the dynamics of tidal sandbanks is of both scientific and practical interest. They provide an attractive option for the extraction of aggregates (Van Lancker et al., 2010), a habitat for marine flora and fauna (Kaiser et al., 2004; Atalah et al., 2013), a foundation for wind farms (Whitehouse et al., 2011; Fairley et al., 2016) and those closer to the coast serve as coastal protection (Dolphin et al., 2007). This wide range of interests emphasizes the importance of understanding the natural dynamics as well as the response to human intervention.

The present study focuses on the natural dynamics, particularly on the plan view evolution of isolated sandbanks. This includes the complex process of bank-breaking, for which two mechanisms have been proposed in the literature (Caston, 1972; Smith, 1988). For the Norfolk Banks, Caston (1972) suggested that an isolated bank breaks into three separate banks (Fig. 1). Alternatively, after examining a kink in the North Hinder Bank, Smith (1988) proposed a mechanism of breaking into two separate banks (Fig. 2). However, both hypotheses were based on interpretation of observations and generic support from a process-based modelling perspective is lacking so far. To clarify this, let us review the literature on sandbank modelling.

Tidal sandbanks have been explained as free instabilities of a flat horizontal sandy seabed subject to an imposed spatially uniform tidal flow (Huthnance, 1982a; De Vriend, 1990; Hulscher et al., 1993). This was shown by linear stability analysis of an idealised morphodynamic model, which typically produces a 'fastest growing mode' showing preferred sandbank spacing and orientation, as well as associated growth (and migration) rates. The instability is driven by horizontal residual circulations resulting from friction- and Coriolis-induced tidetopography interactions, as observed in the field (Caston and Stride, 1970), investigated theoretically (Huthnance, 1973; Zimmerman, 1981; Robinson, 1981; Pattiaratchi and Collins, 1987) and reproduced in complex numerical models (e.g., Sanay et al., 2007). The formation process can also be triggered by a local topographic disturbance, such as a sand extraction pit (Roos et al., 2008). However, these studies are

\footnotetext{
* Corresponding author.

E-mail addresses: thomas.vanveelen@swansea.ac.uk (T.J. van Veelen), p.c.roos@utwente.nl (P.C. Roos), s.j.m.h.hulscher@utwente.nl (S.J.M.H. Hulscher).
} 


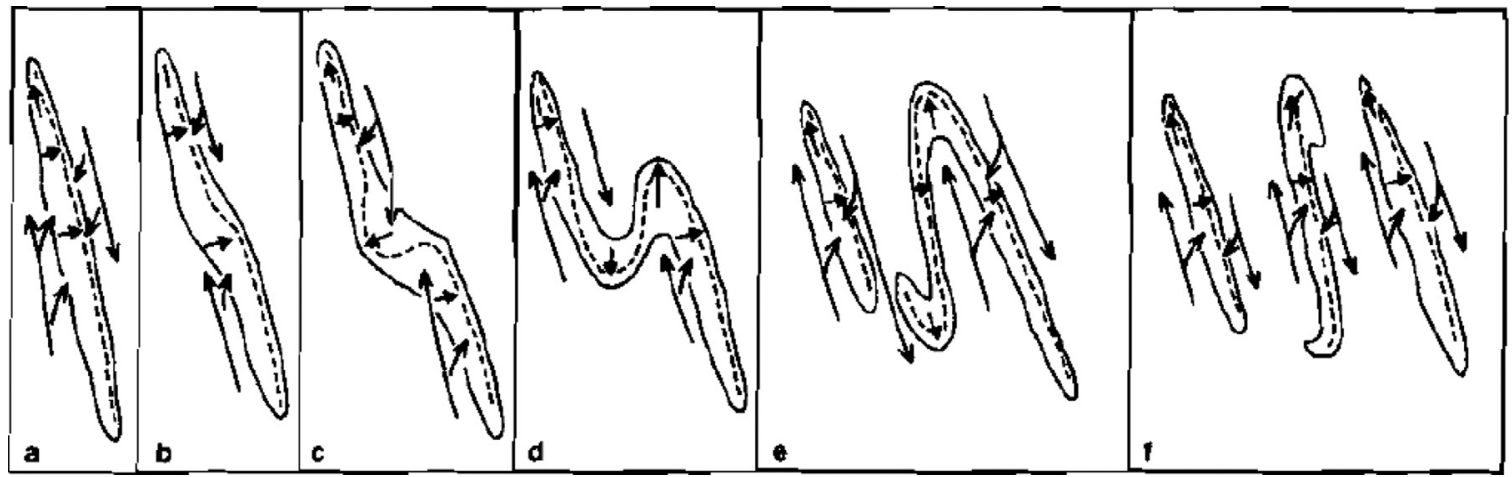

Fig. 1. Breaking mechanism as proposed by Caston (1972): (a) Linear sand body parallel to the direction of the tidal currents, (b) slight "kink" present, possibly due to unequal rate of transport, (c) evolution towards a double curve, (d) the double curve becomes an incipient pair of ebb and flood channels, (e) the channels lengthen, resulting in "blow outs" in the bank, (f) the bank breaks into three parts. The arrows represent the principal tidal flow lines. Reprinted with permission from John Wiley and Sons.

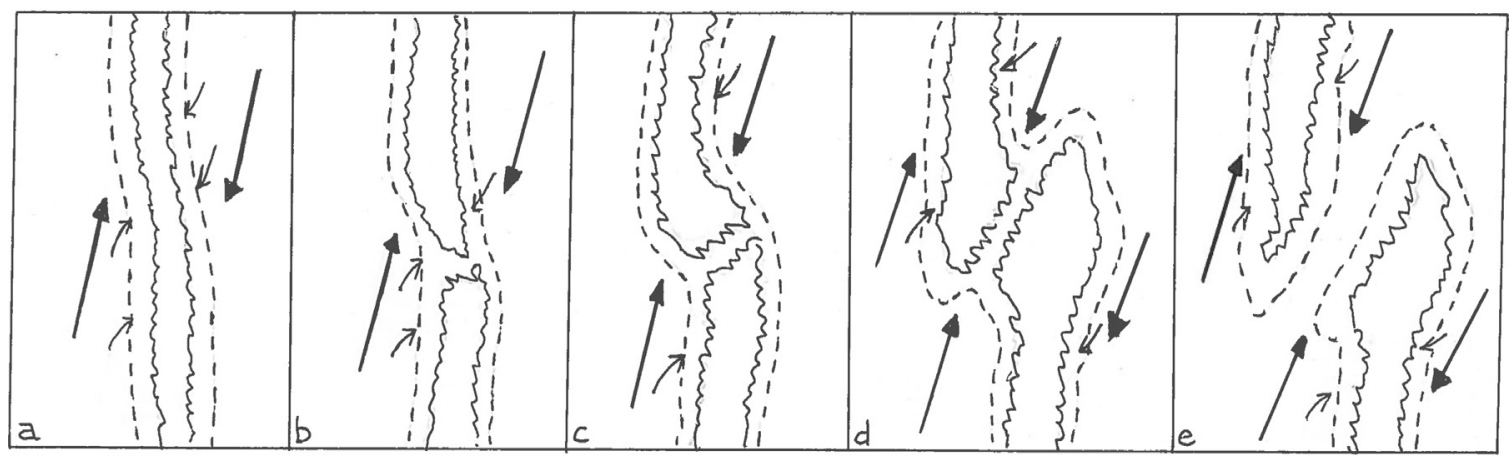

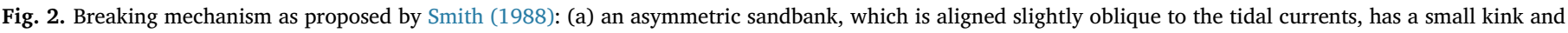

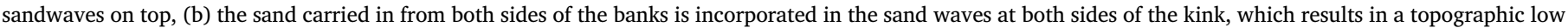

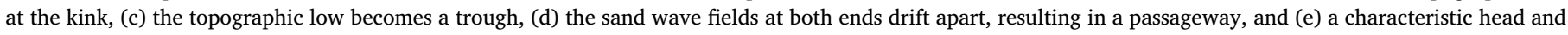
tail, when the banks have broken. The arrows represent the principal tidal flow lines. Image adapted from Smith (1988).

linear in the ratio $\epsilon$ of bank elevation (w.r.t. mean depth) and mean depth and therefore restricted to the initial stage of formation.

To overcome this restriction, the linear models above have been extended to the finite-amplitude regime (i.e., nonlinear in $\epsilon$ ). Huthnance (1982a) and Roos et al. (2004) thus obtained equilibrium profiles, albeit under the assumption of parallel depth contours. Without this restriction, Huthnance (1982b) found bank elongation, evolution towards an S-shape and rotation towards the angle of preferred deposition, but did not report on bank-breaking. Importantly, he used rather simplified hydrodynamics (neglecting inertial and Coriolis terms, and simplifying the tidal cycle into a sequence of two steady flows in opposite directions) and limited sediment availability. Recently, using a two-dimensional numerical model, Yuan et al. (2017) found sandbanks with spatially meandering crests that oscillate in time. Although this was linked to bank-breaking, the applied model domain was too small to study this for an isolated sandbank. Alternatively, for specific forcing conditions (a near-circular tidal ellipse) the linear growth characteristics are such as to enable a weakly nonlinear analysis (Tambroni and Blondeaux, 2008).

In a different class of studies, detailed measurements are combined with site-specific numerical model simulations to investigate how the corresponding sediment transport patterns contribute to bank stability. Examples include Middelkerke Banks (Williams et al., 2000; Pan et al., 2007), Hinder Banks (Deleu et al., 2004), Great Yarmouth Banks (Horrillo-Caraballo and Reeve, 2008) and Kwinte Bank (Brière et al., 2010; Van den Eynde et al., 2010). In particular, Deleu et al. (2004) found support for Smith's bank-breaking hypothesis. However, it proved difficult to apply this class of studies on the time scale of centuries on which sandbanks evolve.
The aim of the present study is to provide a generic process-based modelling framework for the long-term plan view evolution of tidal sandbanks, allowing for interpretation of the bank-breaking mechanisms proposed by Caston (1972) and Smith (1988). To this end, we develop an idealised model, which includes tide-topography interactions, captures long-term nonlinear dynamics and allows for topographies varying in both horizontal directions. Rather than seeking equilibrium profiles, we focus on the transient dynamics of isolated sandbanks. Our interest lies in qualitative behaviour, specifically whether bank-breaking occurs and how this depends on initial bank topography and hydrodynamic settings. Specifically, the innovation of our work lies in the combination of these elements (nonlinear transient dynamics of sandbanks varying in both horizontal directions) as well as our hydrodynamic solution method (applying a truncated expansion in $\epsilon)$.

This paper is organized as follows. First, the model is presented in Section 2, including a scaling procedure and details of the initial bank topography. Next, Section 3 contains the solution procedure, particularly the truncated expansion in $\epsilon$ for the hydrodynamics. The model results, presenting two different cases and a sensitivity analysis, are shown in Section 4. Finally, Sections 5 and 6 contain the discussion and conclusions, respectively.

\section{Model formulation}

\subsection{Geometry}

Consider an offshore region of a shallow shelf sea, far away from coastal boundaries. Hence, influences of a coastline and shelf slope can 


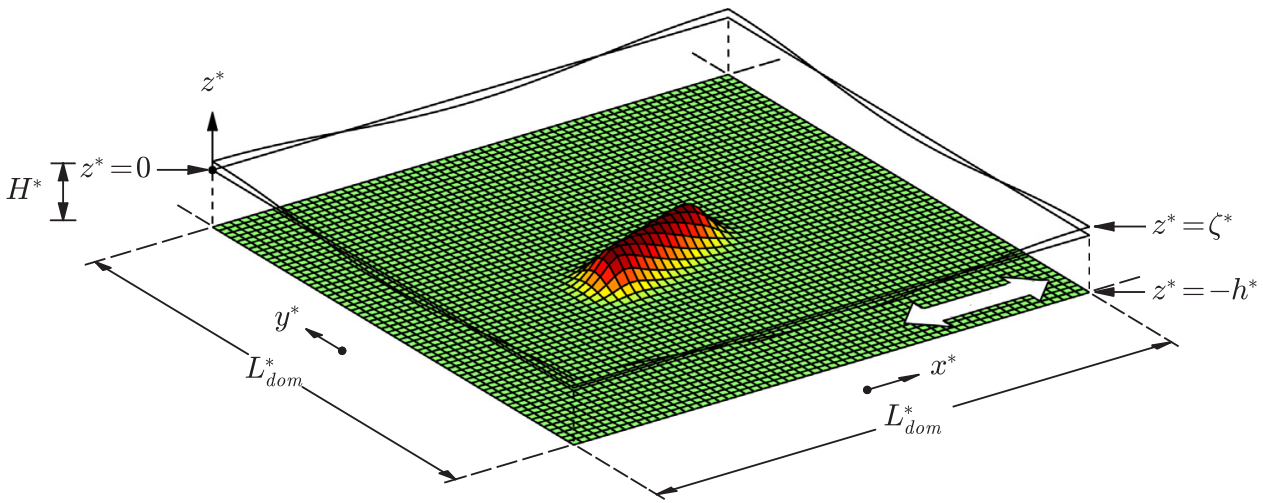

Fig. 3. Definition sketch of the model geometry, showing a spatially periodic domain of dimension $L_{\text {dom }}^{*}$. The ambient water depth is $H^{*}$, the free surface is denoted by $z^{*}=\zeta^{*}$ and the bed level by $z^{*}=-h^{*}$. For details of the initial bank topography, see Section 2.5 and Fig. 4 . The basic flow (flow over a horizontal flat bed) is parallel to the $x$-axis, as denoted by the white double-headed arrow.

(a) top view

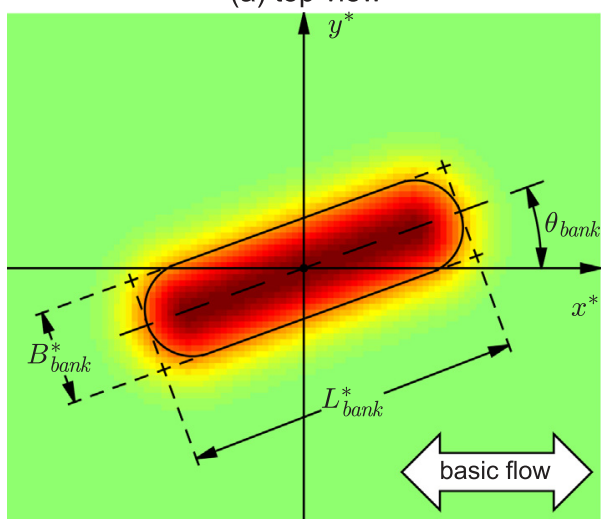

(b) along-bank profile (along central axis)

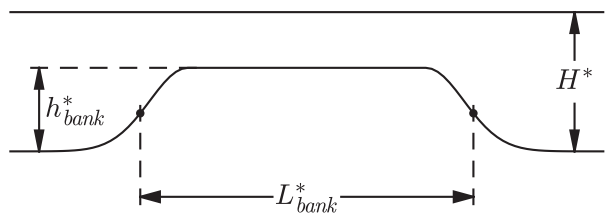

(c) cross-bank profile (in central part)

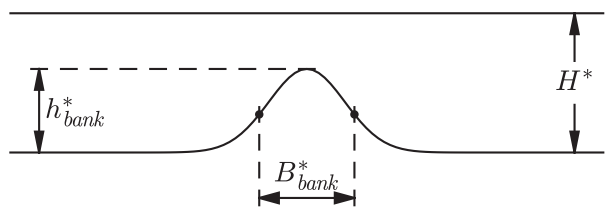

Fig. 4. Definition sketch of the initial bank topography, showing its height $h_{\text {bank }}^{*}$, length $L_{\text {bank }}^{*}$, width $B_{\text {bank }}^{*}$ and orientation $\theta_{\text {bank }}$ : (a) top view, (b) along-bank profile along central axis, (c) cross-bank profile in central part. Bank length and width are reflected in the solid contour, where the height is a fraction exp $(-\pi / 4) \approx 0.46$ of $h_{b a n k}^{*}$. The white double-headed arrow denotes the direction of the basic flow.

be neglected. The ambient water depth $H^{*}$ is in the order of $20-30 \mathrm{~m}$, with an asterisk denoting an unscaled quantity. We adopt a square model domain of length and width $L_{d o m}^{*}$ in the horizontal $x^{*}$ - and $y^{*}$-directions, with spatially periodic boundaries (Fig. 3). With $L_{\text {dom }}^{*}=100 \mathrm{~km}$, our domain size is much larger than the horizontal scales of an individual sandbank (see discussion in Section 5.4). The $z^{*}$-axis points upward, with the free surface elevation at $z^{*}=\zeta^{*}\left(x^{*}, y^{*}, t^{*}\right)$, which averages to $z^{*}=0$. Tidal sandbanks and their evolution are described by a spatiotemporally varying bed level $z^{*}=-h^{*}\left(x^{*}, y^{*}, t^{*}\right)$. A typical example of an initial topography is shown in Fig. 3; further details follow in Section 2.5 and Fig. 4.

\subsection{Model equations}

The model is kept as simple as possible, while still retaining the essential physics. In line with earlier studies, we adopt depth-averaged flow including acceleration, advection, bed friction and the Coriolis effect (e.g., Huthnance, 1982a; Huthnance, 1982b; Hulscher et al., 1993; Roos et al., 2008). The depth-averaged tidal flow velocity $\vec{u}^{*}=\left(u^{*}, v^{*}\right)$ is dominated by a semidiurnal lunar component (M2 with angular frequency $\sigma^{*}=1.405 \times 10^{-4} \mathrm{rad} \mathrm{s}^{-1}$ ) with a maximum flow velocity $U^{*}$, typically $0.5-1.0 \mathrm{~m} \mathrm{~s}^{-1}$. The momentum and mass balances are expressed by the nonlinear depth-averaged shallow water equations, which take the following form:

$\frac{\partial u^{*}}{\partial t^{*}}+u^{*} \frac{\partial u^{*}}{\partial x^{*}}+v^{*} \frac{\partial u^{*}}{\partial y^{*}}-f^{*} v^{*}+\frac{r^{*} u^{*}}{h^{*}+\zeta^{*}}=-F^{*}-g^{*} \frac{\partial \zeta^{*}}{\partial x^{*}}$,

$$
\begin{aligned}
& \frac{\partial v^{*}}{\partial t^{*}}+u^{*} \frac{\partial v^{*}}{\partial x^{*}}+v^{*} \frac{\partial v^{*}}{\partial y^{*}}+f^{*} u^{*}+\frac{r^{*} v^{*}}{h^{*}+\zeta^{*}}=-G^{*}-g^{*} \frac{\partial \zeta^{*}}{\partial y^{*}}, \\
& \frac{\partial h^{*}}{\partial t^{*}}+\frac{\partial \zeta^{*}}{\partial t^{*}}+\frac{\partial}{\partial x^{*}}\left[\left(h^{*}+\zeta^{*}\right) u^{*}\right]+\frac{\partial}{\partial y^{*}}\left[\left(h^{*}+\zeta^{*}\right) v^{*}\right]=0 .
\end{aligned}
$$

Here, $f^{*}=2 \Omega^{*} \sin \varphi$ denotes the Coriolis parameter (with $\Omega^{*}=7.292 \times 10^{-5} \mathrm{rad} \mathrm{s}^{-1}$ the angular frequency of the Earth's rotation and $\varphi$ the latitude). Following Lorentz' linearisation, $r^{*}=\frac{8}{3 \pi} c_{d} U^{*}$ denotes the linear friction coefficient with dimensionless drag coefficient $c_{d}=2.5 \times 10^{-3}$ (e.g., Zimmerman, 1982; Huthnance, 1982a, 1982b; Roos and Hulscher, 2003). Furthermore, $g^{*}=9.81 \mathrm{~m} \mathrm{~s}^{-2}$ is the gravitational acceleration. Finally, $\left(F^{*}, G^{*}\right)$ are forcing terms, representing a spatially uniform yet time-dependent pressure gradient, which, in the case of a flat bed, drives a prescribed tidal flow (details in Section 2.4).

Regarding sediment transport, we include bed load as it is generally considered the dominant transport mode for sandbank dynamics (e.g., Besio et al., 2006). Following earlier studies (e.g., Hulscher et al., 1993; Calvete et al., 2002; Roos et al., 2004), we adopt the following transport formula:

$\vec{q}^{*}=\alpha^{*}\left(\left|\vec{u}^{*}\right|^{2}+\frac{1}{2} U_{w}^{* 2}\left[\frac{h^{*}}{H^{*}}\right]^{-2}\right)\left(\vec{u}^{*}+\lambda^{*} \vec{\nabla}^{*} h^{*}\right)$.

Here, $\vec{q}^{*}=\left(q_{x}^{*}, q_{y}^{*}\right)$ is the wave-averaged sediment transport in $\mathrm{m}^{2} \mathrm{~s}^{-1}$ and $\alpha^{*}$ a proportionality coefficient in $\mathrm{s}^{2} \mathrm{~m}^{-1}$ (Van Rijn, 1993). Wind wave effects are included using a depth-dependent stirring term with reference velocity $U_{w}^{*}$. Furthermore, $\lambda^{*}=\tilde{\lambda}^{*} U^{*}$ is an isotropic bed slope coefficient, with dimensionless $\tilde{\lambda}^{*}$ inversely proportional to the angle of 
repose (Sekine and Parker, 1992), and $\vec{\nabla}^{*}=\left(\frac{\partial}{\partial x^{*}}, \frac{\partial}{\partial y^{*}}\right)$ is the horizontal nabla operator. A discussion of the schematisations in Eq. (4) is given in Section 5.4.

Finally, bed evolution follows from the divergence of the sediment transport according to Exner's equation:

$\left(1-\epsilon_{p o r}\right) \frac{\partial h^{*}}{\partial t^{*}}=\frac{\partial q_{x}^{*}}{\partial x^{*}}+\frac{\partial q_{y}^{*}}{\partial y^{*}}$.

Herein, $\epsilon_{p o r}=0.4$ denotes the porosity of the seabed.

\subsection{Scaling procedure}

We introduce dimensionless coordinates (without asterisk) according to

$(x, y)=\frac{\left(x^{*}, y^{*}\right)}{L^{*}}, \quad z=\frac{z^{*}}{H^{*}}, \quad t=\sigma^{*} t^{*}, \quad \tau=\frac{t^{*}}{T_{m o r}^{*}}$

Herein, the tidal excursion length $L^{*}=U^{*} / \sigma^{*}$ serves as horizontal length scale and the ambient water depth $H^{*}$ serves as vertical length scale. Two time scales are identified to distinguish between dynamics in the short-term (tidal cycle) and long-term (scale of bed evolution). In Eq. (6), we have thus introduced a tidal time coordinate $t$ for the hydrodynamics and sediment transport, and a slower morphological time coordinate $\tau$ for bed evolution, with

$T_{m o r}^{*}=\frac{\left(1-\epsilon_{p o r}\right) H^{*} L^{*}}{\alpha^{*} U^{* 3}}$.

This means that the bed level is a function of $\tau$ but not of $t$, to be justified further below. Note that Eq. (4) was already averaged over the shorter time scale of wind waves.

Next, we introduce scaled quantities according to

$$
\left.\begin{array}{l}
(u, v)=\frac{\left(u^{*}, v^{*}\right)}{U^{*}}, \quad h=\frac{h^{*}}{H^{*}}, \quad \zeta=\frac{g^{*} \zeta^{*}}{U^{* 2}}, \\
(F, G)=\frac{\left(F^{*}, G^{*}\right)}{U^{*} \sigma^{*}}, \quad\left(q_{x}, q_{y}\right)=\frac{\left(q_{x}^{*}, q_{y}^{*}\right)}{\alpha^{*} U^{* 3}} .
\end{array}\right\}
$$

In terms of the scaled coordinates and quantities, the hydrodynamic model Eqs. (1)-(3) become:

$\frac{\partial u}{\partial t}+u \frac{\partial u}{\partial x}+v \frac{\partial u}{\partial y}-f v+\frac{r u}{h}=-F-\frac{\partial \zeta}{\partial x}$,

$\frac{\partial v}{\partial t}+u \frac{\partial v}{\partial x}+v \frac{\partial v}{\partial y}+f u+\frac{r v}{h}=-G-\frac{\partial \zeta}{\partial y}$,

$\frac{\partial(h u)}{\partial x}+\frac{\partial(h v)}{\partial y}=0$.

Herein, we have introduced scaled Coriolis and friction coefficients $f=f^{*} / \sigma^{*}$ and $r=r^{*} /\left(\sigma^{*} H^{*}\right)$, respectively. The dimensionless forcing $(F, G)$ is specified in Section 2.4. Furthermore, in Eqs. (9)-(11) we have applied the rigid lid assumption. This means that the contribution of the free surface elevation to the mean water depth is neglected, which is justified by the small value of the squared Froude number $\mathrm{Fr}^{2}=U^{* 2} /\left(\mathrm{g}^{*} H^{*}\right) \approx 10^{-3}$. Further, because $\gamma=\left(\sigma^{*} T_{\text {mor }}^{*}\right)^{-1} \approx 10^{-6}$, bed level fluctuations on the tidal time scale can indeed be neglected. This justifies the decoupling of the hydrodynamic equations from the sediment transport and the bed evolution, which is commonly known as the quasi-stationary approach.

Next, the scaled version of the sediment transport formula Eq. (4) reads

$\vec{q}=\left(|\vec{u}|^{2}+\frac{1}{2} U_{w}^{2} h^{-2}\right)(\vec{u}+\lambda \vec{\nabla} h)$.

Herein, we use the scaled stirring velocity $U_{w}=U_{w}^{*} / U^{*}$ and the scaled bed slope coefficient $\lambda=\lambda^{*} H^{*} /\left(L^{*} U^{*}\right)$.

Finally, the scaled version of Exner's Eq. (5) is given by $\frac{\partial h}{\partial \tau}=\frac{\partial\left\langle q_{x}\right\rangle}{\partial x}+\frac{\partial\left\langle q_{y}\right\rangle}{\partial y}$.

The angle brackets denote averaging over a tidal cycle: $\langle\cdot\rangle=\frac{1}{2 \pi} \int_{0}^{2 \pi} \cdot \mathrm{d} t$. Because of the quasi-stationary approach, only the tidally averaged sediment flux effectively contributes to the bed evolution.

\subsection{Tidal forcing}

The tidal forcing represents a spatially uniform yet time-dependent pressure gradient, which reads

$(F, G)=\mathfrak{R}\{(\widehat{F}, \widehat{G}) \exp (i t)\}$.

The complex Fourier coefficients $(\widehat{F}, \widehat{G})$ are chosen such that, in the case of a flat bed, a prescribed M2-tidal flow is generated:

$(u, v)=(\cos t, 0)=\mathfrak{R}\{(1,0) \exp (i t)\}$.

This prescribed tidal flow, in stability analysis referred to as 'basic flow' (e.g., see Section 3.4), describes a unidirectional oscillation parallel to the $x$-axis.

Expressions for the complex coefficients $(\widehat{F}, \widehat{G})$ are now obtained by substituting Eqs. (14)-(15) into the scaled momentum Eqs. (9)-(10), with omission of the horizontal derivatives, i.e. applying $\frac{\partial}{\partial x}=\frac{\partial}{\partial y}=0$ :

$(\widehat{F}, \widehat{G})=-(i+r / h, f)$.

The tidal forcing in Eq. (14) can be easily extended such that the basic flow includes residual currents ('M0', in arbitrary direction), an M2ellipse (with arbitrary amplitude, ellipticity, phase and inclination in the horizontal plane) and tidal asymmetry (e.g., by also adding an M4ellipse). Importantly, as will be clarified in Section 3, our model captures the tide-topography interactions that generate the residual currents and higher harmonics in the presence of a sandbank.

\subsection{Initial topography}

The initial topography in this study consists of a single isolated sandbank located in the middle of an otherwise flat domain; see the three-dimensional impression in Fig. 3. As further shown in Fig. 4, it is characterised by its height $h_{\text {bank }}^{*}$, length $L_{\text {bank }}^{*}$, width $B_{\text {bank }}^{*}$ and orientation $\theta_{\text {bank }}$ with respect to the principal direction of the tidal flow (being the $x^{*}$-axis). The central part of the bank is uniform in along-bank direction and Gaussian in the cross-bank direction, whereas the bank ends are Gaussian in two-dimensional sense. The bank height $h_{\text {bank }}^{*}$ is thus attained along the bank's axis in the central part.

Expressions for the bank topography are given in Appendix A. The bank parameters are defined such that the bank volume is simply the product of height, length and width: $V_{b a n k}^{*}=h_{b a n k}^{*} L_{b a n k}^{*} B_{b a n k}^{*}$.

\section{Solution procedure}

\subsection{Outline}

The solution procedure closely follows the morphological loop, as outlined in Fig. 5. We first write the topography as the sum of a spatially uniform $h_{0}$ and a spatially varying part $\epsilon h_{1}$, thus introducing a dimensionless expansion parameter $\epsilon$ (details in Section 3.2). Next, we present a compact formulation of the hydrodynamic problem in terms of the vorticity $\eta$ (Section 3.3). We then define the hydrodynamic solution vector

$\phi=(\eta, u, v, \zeta)$

which is expanded in powers of $\epsilon$ and solved from a sequence of linear problems (Section 3.4). Finally, the sediment fluxes are obtained directly from the bed load transport formulation from the hydrodynamic solution and, based on the tide-averaged sediment fluxes, bed evolution is computed via a fourth order Runge-Kutta scheme (Section 3.5). This 


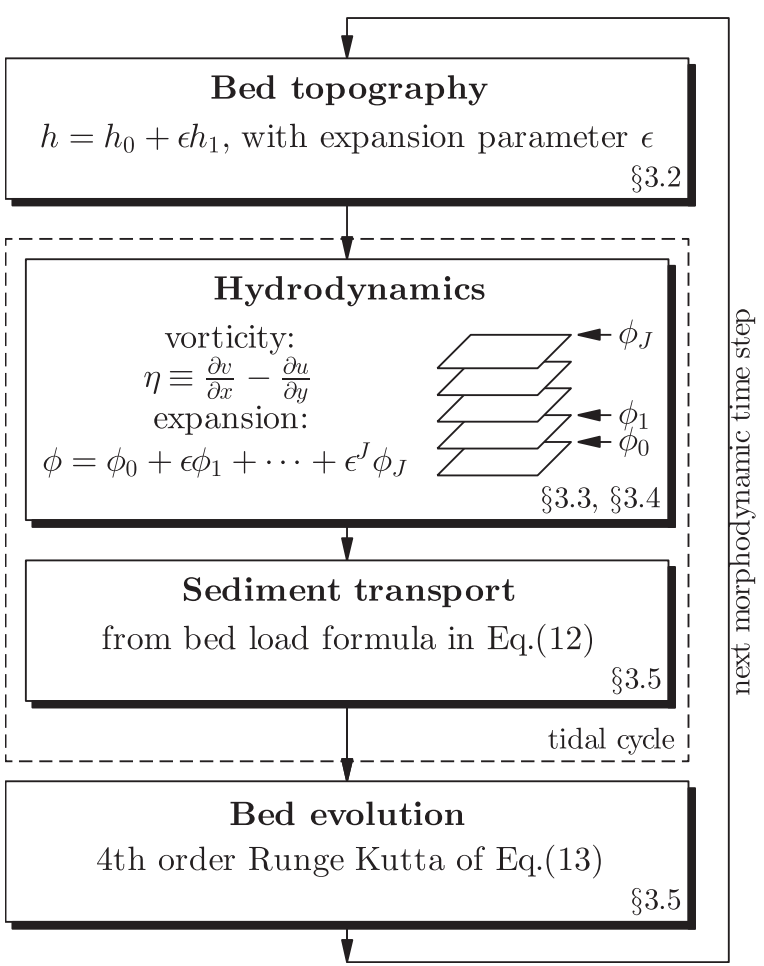

Fig. 5. Schematic of the solution procedure, which follows the morphological loop. For an explanation, see Section 3.1.

closes the loop and the updated bed topography serves as input for the next morphodynamic time step.

Additionally, to enable a comparison between linear and nonlinear dynamics, we also present a model solution that is linear in $\epsilon$, i.e. with linear hydrodynamics and linearised sediment transport (Section 3.6).

\subsection{Perturbed topography}

The topography $h(x, y, \tau)$ is written as the sum of the mean water depth $h_{0}$ and a spatially varying perturbed topography $h_{1}(x, y, \tau)$ (with vanishing spatial average) multiplied by a dimensionless expansion parameter $\epsilon$ :

$h=h_{0}+\epsilon h_{1}$.

Because the sandbank in our spatially periodic domain is purely of elevation, the mean water depth (scaled against ambient depth) is slightly smaller than one: $h_{0}=1-V_{\text {bank }}^{*} /\left(H^{*} L_{\text {dom }}^{* 2}\right)<1$. The expansion parameter $\epsilon$ is positive and defined such that $h_{1}$ is of order one, i.e. $\max _{x, y}\left|h_{1}(x, y, \tau)\right|=1$. This implies

$\epsilon=\frac{\max _{x^{*}, y^{*}}\left|h^{*}-H_{\text {mean }}^{*}\right|}{H_{\text {mean }}^{*}} \approx \frac{h_{\text {bank }}^{*}}{H^{*}}$,

where $H_{\text {mean }}^{*}=H^{*} h_{0}$ is the unscaled mean (not ambient) depth. The second statement in Eq. (19) is an approximation and not an equality, because, as noted above, $H_{\text {mean }}^{*}$ is slightly smaller than $H^{*}$ (i.e., $h_{0}$ is slightly smaller than 1 ). Unlike linear stability analysis, where $\epsilon \ll 1$, we now allow for larger values of $\epsilon$.

The perturbed topography is written as a truncated Fourier series

$h_{1}=\sum_{m=-M}^{M} \sum_{n=-M}^{M} \hat{h}_{1 m n}(\tau) \exp \left(i\left[k_{m} x+l_{n} y\right]\right)$.

Here, we have introduced the complex Fourier coefficients $\hat{h}_{1 m n}(\tau)$, defined for the topographic wave numbers $\left(k_{m}, l_{n}\right)=(m, n) k_{\min }$, in which $k_{\min }=2 \pi L^{*} / L_{d o m}^{*}$ is the minimum wave number associated with the domain size. This formulation satisfies the periodic boundaries.
Furthermore, $M$ is the spatial truncation number, similar for both horizontal directions so as to avoid any directional preference in our square model domain. In our simulations (see Section 4), we set $L_{\text {dom }}^{*}=100 \mathrm{~km}$ and $M=128$.

Finally, we note that Eq. (20) in fact projects the sandbank topography onto the eigenmodes of the linear problem, as has been applied earlier in linear model studies of, e.g., sandpits (Roos et al., 2005; Roos et al., 2008) and shoreface nourishments (van Leeuwen et al., 2007).

\subsection{Vorticity}

It is convenient to solve the spatially varying part of the momentum balance in terms of the vorticity $\eta$, which, in dimensionless form, is defined as

$\eta \equiv \frac{\partial v}{\partial x}-\frac{\partial u}{\partial y}$.

An evolution equation for this quantity is obtained by cross-differentiation of the momentum Eqs. (9)-(10) and application of the continuity Eq. (11). This leads to

$\mathscr{L} \eta=\underbrace{\left[\frac{f}{h} \frac{\partial h}{\partial x}-\frac{r}{h^{2}} \frac{\partial h}{\partial y}\right]}_{R} u+\underbrace{\left[\frac{f}{h} \frac{\partial h}{\partial y}+\frac{r}{h^{2}} \frac{\partial h}{\partial x}\right]}_{S} v$,

with operator $\mathscr{L}=\frac{\partial}{\partial t}+u \frac{\partial}{\partial x}+v \frac{\partial}{\partial y}+r / h$. The vorticity thus experiences acceleration, advection and dissipation due to bottom friction (lefthand side), and it is produced by Coriolis and frictionally induced tidetopography interactions (contained in the terms $R u$ and $S v$ on the righthand side).

Because the above formulation in terms of vorticity does not capture the spatially uniform part of the flow, we must supplement the vorticity Eq. (22) with spatially averaged versions of the momentum Eqs. (9)-(10).

\subsection{Hydrodynamic solution}

The hydrodynamic solution $\phi=(\eta, u, v, \zeta)$ is expanded in powers of $\epsilon$ according to

$\phi=\sum_{j=0}^{J} \epsilon^{j} \phi_{j}=\phi_{0}+\epsilon \phi_{1}+\epsilon^{2} \phi_{2}+\cdots+\epsilon^{J} \phi_{J}$.

We thus distinguish contributions $\phi_{0}, \phi_{1}$ and $\phi_{j}$ for $j \geq 2$, which represent, respectively, the basic flow (over a flat bed, see Section 2.4), first order flow (linear response) and higher order flow solutions (nonlinear response). We truncate the hydrodynamic expansion when the estimations of the functions $R$ and $S$ in Eq. (22) deviate less than $10^{-5}$ from the exact value, with a maximum of $J=10$.

We proceed by substitution of our expansion in Eq. (23) into the vorticity Eq. (22) and collecting like powers of $\epsilon$. Implicit herein is the assumption that each of the dimensionless parameters $\mathrm{Fr}^{2}$ and $\gamma$ (see Section 2.3) is smaller than $\epsilon^{j}$ for any $j=1,2, \cdots, J$. Thus, at each order $j$, the vorticity $\eta_{j}$ must satisfy a linear problem:

$\mathscr{L}_{0} \eta_{j}=b_{j}$.

Herein, $\mathscr{L}_{0}=\frac{\partial}{\partial t}+u_{0} \frac{\partial}{\partial x}+v_{0} \frac{\partial}{\partial y}+r / h_{0}$ is now a linear operator, because the lowest order flow $\left(u_{0}, v_{0}\right)$ and topography $h_{0}$ are all known. As it turns out, the right-hand side $b_{j}$ is a function of lower order flow solutions (involving $\phi_{0}, \phi_{1}$ up to $\phi_{j-1}$ ). With the basic flow prescribed, this property enables us to first calculate the linear flow solution (details in Appendix B) and then the nonlinear flow solutions at subsequent orders $j \geq 2$ (Appendix C).

As already noted in Section 3.3, solving for the spatially uniform part of the $j$-th order flow requires considering the spatially averaged versions of the momentum Eqs. (9)-(10). 


\subsection{Sediment transport and bed evolution}

Once the hydrodynamic solution is known up to a prescribed order $J$, we apply Eq. (23) and use the truncated flow velocity vector $\vec{u}=(u, v)=\sum_{j=0}^{J} \epsilon^{j}\left(u_{j}, v_{j}\right)$ as direct input for the sediment transport formula (12). This yields $\vec{q}$ and, after transforming to Fourier space, $\left(\hat{q}_{x, m n}, \hat{q}_{y, m n}\right)$ for each Fourier mode $(m, n)$.

Exner's Eq. (13) is then applied to obtain an expression for the bed evolution in Fourier space, which is based on the tidally averaged sediment fluxes according to

$\frac{\partial \hat{h}_{1 m n}}{\partial \tau}=i k_{m}\left\langle\hat{q}_{x, m n}\right\rangle+i l_{n}\left\langle\hat{q}_{y, m n}\right\rangle$.

The tidal averaging is carried out numerically over $N_{\text {tide }}$ points in the tidal cycle. Morphodynamic time-stepping is conducted via the fourth order Runge-Kutta method, with time step $\Delta \tau$. This update of the bed topography effectively closes the morphodynamic loop, as depicted in Fig. 5.

\subsection{Model solution with linear dynamics}

To enable a comparison between linear and nonlinear dynamics, here we also present a model version with linear hydrodynamics and linearised sediment transport (e.g., Hulscher et al., 1993). This requires setting $J=1$ in our hydrodynamic expansion in Eq. (23) and replacing the sediment transport formula (12) with its linearised counterpart. This implies $\vec{q}=\overrightarrow{q_{0}}+\epsilon \overrightarrow{q_{1}}$, in which

$\overrightarrow{q_{1}}=\left(\left|\overrightarrow{u_{0}}\right|^{2}+\frac{1}{2} U_{w}^{2} h_{0}^{-2}\right)\left(\overrightarrow{u_{1}}+\lambda \vec{\nabla} h_{1}\right)+\left(2\left(\overrightarrow{u_{0}} \cdot \overrightarrow{u_{1}}\right)-U_{w}^{2} h_{0}^{-3} h_{1}\right) \overrightarrow{u_{0}}$

According to linear analysis, the individual spatial modes experience exponential growth or decay according to

$\hat{h}_{1 m n}(\tau)=\hat{h}_{1 m n}^{\text {init }} \exp \left(\omega_{m n} \tau\right)$,

with initial amplitude $h_{1 m n}^{\text {init }}$ and growth rate $\omega_{m n}$. Because in this study we consider symmetric forcing only (i.e., by a single tidal consituent), the growth rate is real-valued. Allowing for asymmetries in the forcing would trigger migration, implying a nonzero imaginary part of $\omega_{m n}$ (also see Section 2.4).

As already pointed out in the Introduction, the most important result of a linear stability analysis is the 'fastest growing mode', i.e. the topographic wave vector $\overrightarrow{k_{f g m}}=\left(k_{f g m}, l_{f g m}\right)$ for which the real part of the growth rate $\omega_{f g m}$ attains its maximum. The crest orientation $\theta_{f g m}=90^{\circ}+\tan ^{-1}\left(l_{f g m} / k_{f g m}\right)$, wavelength $\lambda_{f g m}^{*}=2 \pi L^{*} /\left|\overrightarrow{k_{f g m}}\right|$ and efolding growth time $T_{f g m}^{*}=T_{m o r}^{*} / \omega_{f g m}$ associated with this fastest growing mode will be used when presenting and interpreting the model results.

\section{Results}

\subsection{Overview of simulations}

Assuming typical North Sea conditions, we present two cases A and B that display qualitatively different plan view evolutions. The cases only differ in their initial topography (Table 1); all physical and numerical parameters are equal (Table 2). For these conditions, the fastest growing mode from linear stability analysis has an orientation $\theta_{\text {fgm }}=44^{\circ}$, a wavelength $\lambda_{f g m}^{*}=7.5 \mathrm{~km}$ and an e-folding growth time of $T_{f g m}^{*} \approx 560$ yrs (Table 2). In conjunction with these cases, we present a sensitivity analysis in which the initial topography and other parameters are systematically varied. Based on these simulations we present a classification scheme of bank evolution and an associated regime diagram.
Table 1

Bank parameters for cases A and B and sensitivity analysis.

\begin{tabular}{|c|c|c|c|c|c|}
\hline Bank parameter $^{\mathrm{a}}$ & Symbol & A & B & Range $^{b}$ & Unit \\
\hline Height $^{c}$ & $h_{\text {bank }}^{*}$ & 4 & 4 & -4 to 12 & $\mathrm{~m}$ \\
\hline Length & $L_{b a n k}^{*}$ & 20 & 10 & $10-40$ & $\mathrm{~km}$ \\
\hline Width & $B_{\text {bank }}^{*}$ & 2 & 2 & $1-4$ & $\mathrm{~km}$ \\
\hline Orientation & $\theta_{\text {bank }}$ & 0 & 44 & -90 to 90 & $\circ$ \\
\hline Expansion parameter ${ }^{\mathrm{d}}$ & $\epsilon$ & 0.16 & 0.16 & $0.16-0.48$ & - \\
\hline
\end{tabular}

a See Section 2.5 .

b For sensitivity analysis in section 4.4 .

c Negative value implies pit instead of bank.

d Initial value of $\epsilon$ in mean water depth $H^{*}=25 \mathrm{~m}$ (Table 2).

Table 2

Physical and numerical model parameters and fgm-properties.

\begin{tabular}{|c|c|c|c|}
\hline Model parameter & Symbol & Value & Unit \\
\hline Ambient water depth & $H^{*}$ & 25 & $\mathrm{~m}$ \\
\hline Latitude & $\varphi$ & 51 & ${ }^{\circ} \mathrm{N}$ \\
\hline Bottom drag coefficient & $c_{d}$ & $2.5 \times 10^{-3}$ & - \\
\hline Sediment transport coefficient & $\alpha^{*}$ & $4.0 \times 10^{-5}$ & $\mathrm{~s}^{2} \mathrm{~m}^{-1}$ \\
\hline Bed slope coefficient & $\tilde{\lambda}^{*}$ & 2.0 & - \\
\hline Wave stirring velocity & $U_{w}^{*}$ & 0.6 & $\mathrm{~m} \mathrm{~s}^{-1}$ \\
\hline Bed porosity & $\epsilon_{\text {por }}$ & 0.4 & - \\
\hline Angular frequency of M2-tide & $\sigma^{*}$ & $1.405 \times 10^{-4}$ & $\operatorname{rad~s}^{-1}$ \\
\hline M2-tidal velocity amplitude & $U^{*}$ & 0.6 & $\mathrm{~m} \mathrm{~s}^{-1}$ \\
\hline Gravitational acceleration & $g^{*}$ & 9.81 & $\mathrm{~m} \mathrm{~s}^{-2}$ \\
\hline Angular frequency of Earth rotation & $\Omega^{*}$ & $7.292 \times 10^{-5}$ & $\mathrm{rad} \mathrm{s}^{-1}$ \\
\hline Tidal excursion length & $L^{*}$ & 5.0 & $\mathrm{~km}$ \\
\hline Morphodynamic time scale & $T_{m o r}^{*}$ & $2.3 \times 10^{2}$ & yrs \\
\hline Coriolis parameter & $f$ & 0.81 & - \\
\hline Bottom friction parameter & $r$ & 0.36 & - \\
\hline Scaled stirring velocity & $U_{w}$ & 1.0 & - \\
\hline Bed slope coefficient & $\lambda$ & 0.012 & - \\
\hline Squared Froude number & $\mathrm{Fr}^{2}$ & $1.5 \times 10^{-3}$ & - \\
\hline Ratio of time scales & $\gamma$ & $9.7 \times 10^{-7}$ & - \\
\hline Domain size & $L_{\text {dom }}^{*}$ & 100 & $\mathrm{~km}$ \\
\hline Expansion truncation number & $J$ & 10 & - \\
\hline Spatial truncation number & $M$ & 128 & - \\
\hline Temporal truncation number ${ }^{e}$ & $P$ & 5 & - \\
\hline Number of points in tide-average & $N_{\text {tide }}$ & 64 & - \\
\hline Morphodynamic time step & $\Delta \tau$ & 0.01 & - \\
\hline Preferred bank orientation ${ }^{f}$ & $\theta_{f g m}$ & 44 & $\circ$ \\
\hline Preferred bank wavelength ${ }^{\mathrm{f}}$ & $\lambda_{\text {fgm }}^{*}$ & 7.5 & $\mathrm{~km}$ \\
\hline e-folding growth time $\mathrm{f}^{\mathrm{f}}$ & $T_{f g m}^{*}$ & $5.6 \times 10^{2}$ & yrs \\
\hline
\end{tabular}

e See Fourier expansion in Eq. (B.3) of Appendix B.

f Corresponding to the fastest growing mode from linear stability analysis; see Section 3.6.

\subsection{Case $A$}

Plan view evolution Case A is a bank parallel to the tidal flow. This choice resembles the initial stage in (Caston, 1972, see Fig. 1). The evolution according to our model is shown in Fig. 6, where for clarity only the central part of the model domain is plotted. We distinguish four characteristic stages I-IV (top row of Fig. 6):

I. The initial topography consists of a bank height of $4 \mathrm{~m}$, bank length of $20 \mathrm{~km}$, bank width of $2 \mathrm{~km}$ and an orientation parallel to the principal tidal flow. In a mean water depth of $H^{*}=25 \mathrm{~m}$, this bank height corresponds to $\epsilon=0.16$.

II. The ends rotate anticlockwise toward the preferred orientation $\theta_{f g m}$ from linear stability analysis. Furthermore, the bed parallel to the head and the tail deepens, which reflects the formation of parallel 

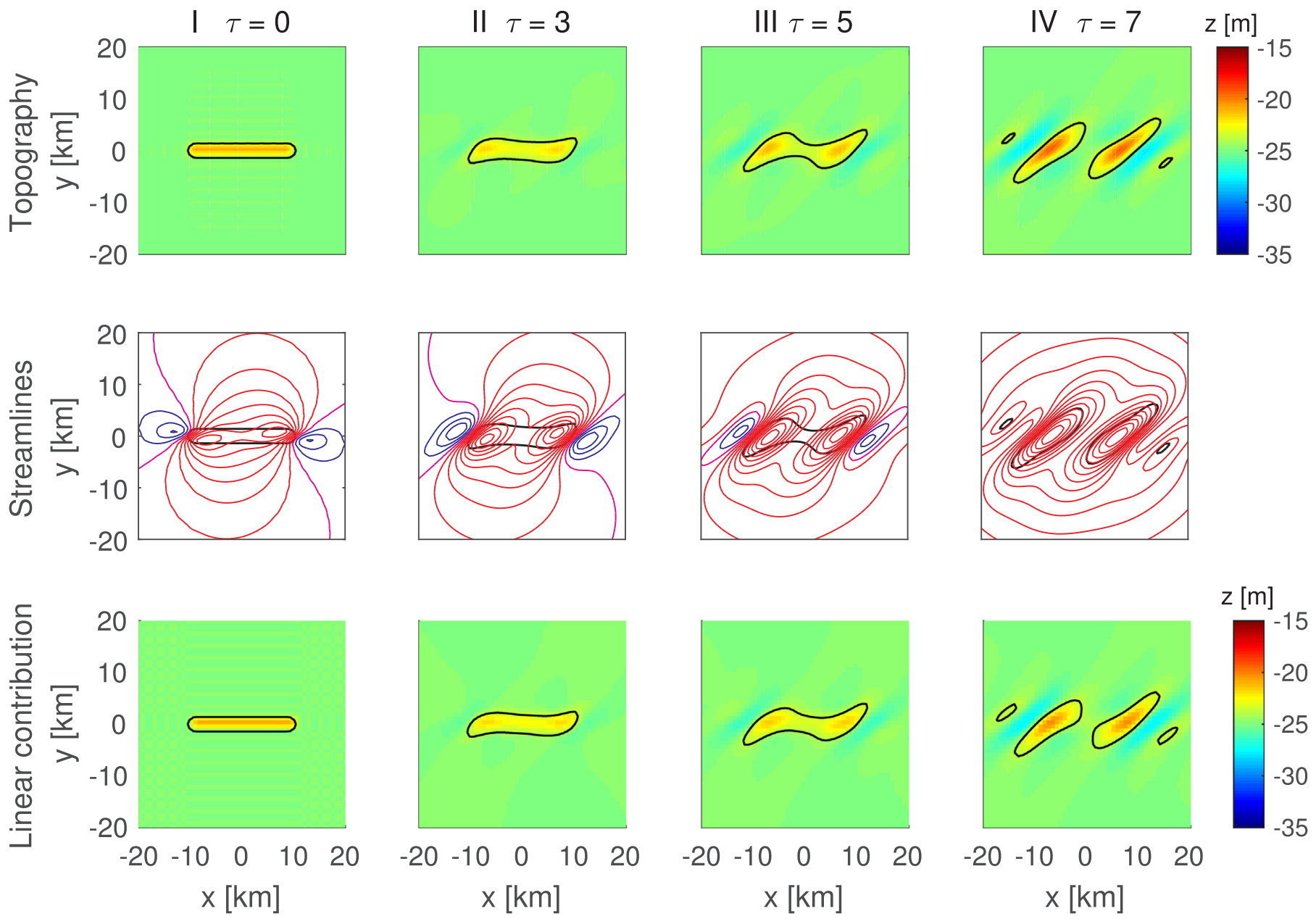

Fig. 6. Evolution of case A at four stages: (I) $\tau=0$, (II) $\tau=3$, (III) $\tau=5$ and (IV) $\tau=7$ with every $\tau$-unit representing $T_{\text {mor }}^{*} \approx 230$ yrs. The top row shows the plan view evolution, the middle row the streamlines $\Psi$ of the depth-integrated residual flow (red clockwise, blue anticlockwise and pink separating the two senses of orientation), and the bottom row the linear contribution (Section 3.6). Note that the actual model domain (100 km) is much larger than the domain plotted here $(40 \mathrm{~km})$. Black lines indicate the $24 \mathrm{~m}$-depth contour.

troughs and banks, i.e. pattern expansion. We also find erosion over the entire bank, especially at the central part. This results in a topography with rotated crests at both bank ends and a slightly lower central part.

III. The elevated crests grow vertically and expand horizontally, while the central part continues to erode. This results in two features separated by a depression.

IV. Further erosion turns the central depression into a trough, which breaks the original bank in two parts. The separate parts now form a patch of parallel banks further elongating and growing in amplitude.

Hydrodynamics The residual circulation associated with each evolutionary stage is shown in the middle row of Fig. 6 . The streamlines are contours of a tide-averaged stream function $\langle\Psi\rangle$, which satisfies

$\frac{\partial \Psi}{\partial y}=h u, \quad \frac{\partial \Psi}{\partial x}=-h v$.

As before, angle brackets $\langle\cdot\rangle$ denote tidal averaging.

At all stages, the pattern is dominated by a clockwise residual circulation (red) around the bank(s), which closely reflects topography. Zones of weak counterclockwise circulation (blue) occur at the bank ends. Also visible is the Coriolis-induced asymmetry with respect to the bank axis. Furthermore, streamlines converge wherever the gradient in topography is perpendicular to the angle of preferred bank orientation. This is where morphodynamic changes are strongest. Finally, bank elongation occurs where streamlines diverge.

Linear contribution The linear solution, as presented in Section 3.6 and shown in the bottom row of Fig. 6, is discussed in Section 5.1.

\subsection{Case $B$}

Plan view evolution The initial bank of case B has an anticlockwise orientation (with respect to the principal tidal flow) and is shorter than the one in case A. This initial topography corresponds to the cases studied in Huthnance (1982b) and (Smith, 1988, see Fig. 2). In the plan view evolution, we again distinguish four stages (top row of Fig. 7):

I. The initial topography consists of a bank with a height of $4 \mathrm{~m}$, length of $10 \mathrm{~km}$, width of $2 \mathrm{~km}$ and an orientation of 44 degrees anticlockwise with respect to the principal tidal current. This angle equals that of the fastest growing mode in the linear model (Section 3.6).

II. The bank axis remains straight, and the central part of the bank grows faster than the bank ends. We also observe the onset of pattern expansion in the form of parallel troughs adjacent to the bank.

III. The bank grows in amplitude and elongates. The fastest growth is observed at the central part of the bank. Further pattern expansion is suppressed, whereas the crest starts to meander.

IV. The central part of the bank aligns toward an orientation parallel to the principal tidal flow, while the ends remain oriented towards the 

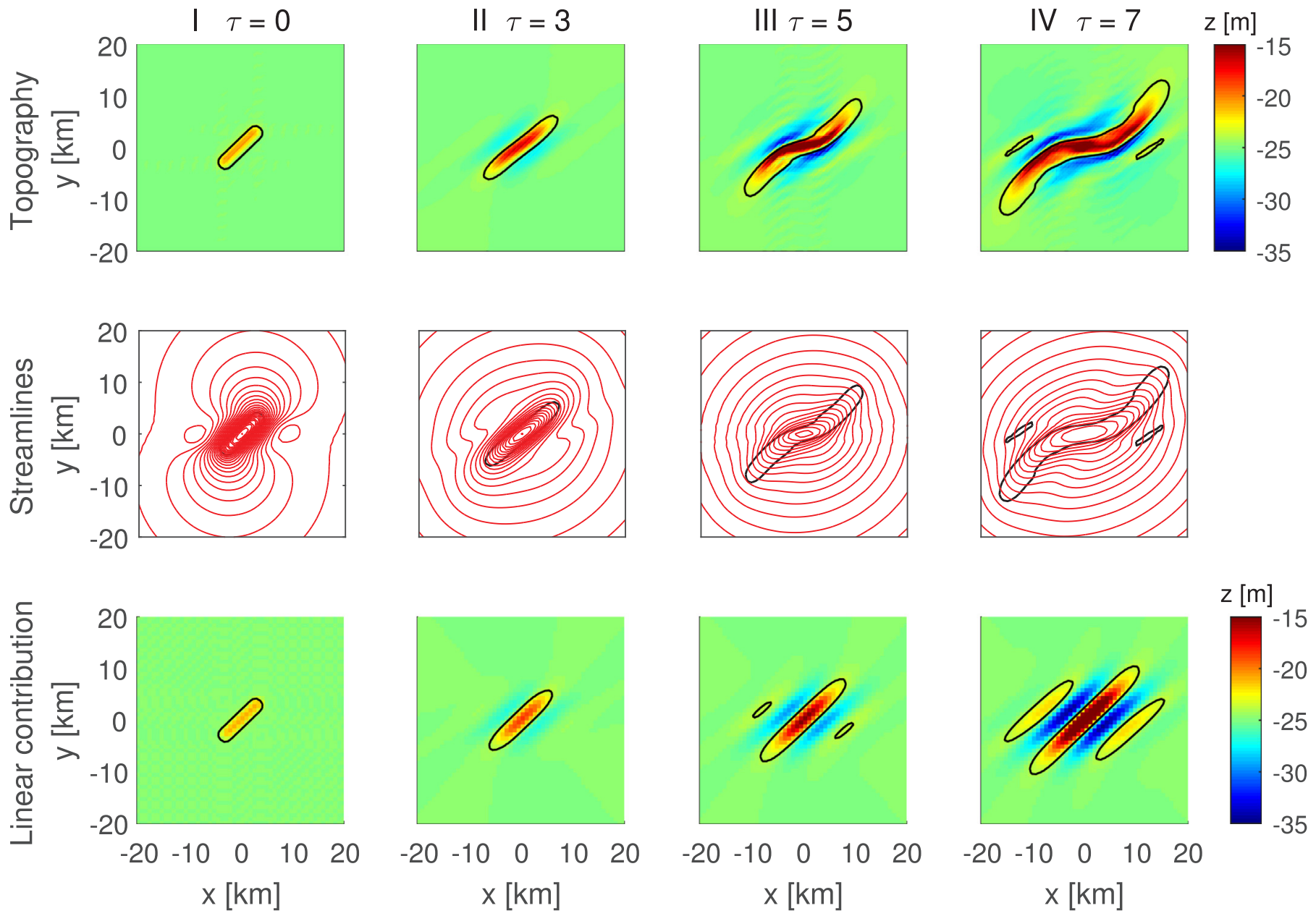

Fig. 7. Evolution of case B at four stages: (I) $\tau=0$, (II) $\tau=3$, (III) $\tau=5$ and (IV) $\tau=7$. For explanation, see caption of Fig. 6 .

preferred bank orientation from linear analysis. Instead of breaking, the bank now attains a pronounced S-shape (or, phrased more accurately, the mirror image of an S-shape).

Hydrodynamics The initial residual circulation pattern (left-hand middle row plot in Fig. 7) resembles that of case A. However, as the banks evolve differently, also the circulations develop into different patterns. In case B, this leads to a clockwise rotation with an almost circular shape in the three subsequent stages.

Linear contribution The linear solution, as presented in Section 3.6 and shown in the bottom row of Fig. 7, is discussed in Section 5.1.

\subsection{Sensitivity to initial topography: Classification scheme and regime diagram}

The distinct evolution of cases A and B shows that changing the initial topography may lead to qualitatively different behaviour. Here, a generalisation of bank dynamics within our full parameter space of the initial topography is presented, keeping flow conditions equal to the cases A and B described previously. Specifically, we varied all four characteristics of the initial topography: bank height, length, width and orientation (parameter ranges in Table 1).

Based on our sensitivity analysis, we identify two types of bank dynamics: banks that break and banks that attain an S-shape. Within each category, the behaviour is remarkably similar. Based on this, we present a classification scheme with two paths A and B (see Fig. 8, distinguishing the four stages I-IV already introduced in Figs. 6 and 7):
Path A: Bank Breaking Starting from an initially straight bank, the ends rotate toward the angle of the fastest growing mode from linear analysis. Additionally, pattern expansion occurs. The bank ends grow faster than the central part of the bank. What follows is a central depression, which forms the onset of bank breaking. Case A, as presented in Section 4.2 and Fig. 6, is a typical example.

Path B: S-shape This path describes banks that do not break, but develop an S-shape. The initial bank retains its shape in the early stages and expands its pattern with parallel troughs and crests. Meanwhile, the central part grows and during this process its preferred angle changes to a direction parallel to the principal tidal flow. As the central part adjusts, an Sshape is created. Case B, as presented in Section 4.3 and Fig. 7, is a typical example.

As it turns out, bank orientation and length are the main drivers for path selection, which can be visualised in a so-called regime diagram (see Fig. 9). Bank height and width affect evolution within a path. The sensitivities are described below.

The bank orientation $\theta_{\text {bank }}$ controls to what extent the bank ends can rotate. If it is close to the preferred orientation from linear stability analysis, i.e. $\theta_{\text {bank }} \approx \theta_{\text {fgm }}$, bank ends will not separate themselves from the main axis. In this case, the bank remains straight and path $B$ will be followed. Alternatively, a bank orientation away from $\theta_{f g m}$ leads to bank-breaking. The deviation from the fastest growing required for bank breaking depends on bank length $L_{b a n k}^{*}$. Shorter banks require a stronger deviation to break than long banks (Fig. 9). Bank length further determines if the bank ends are sufficiently separated for 


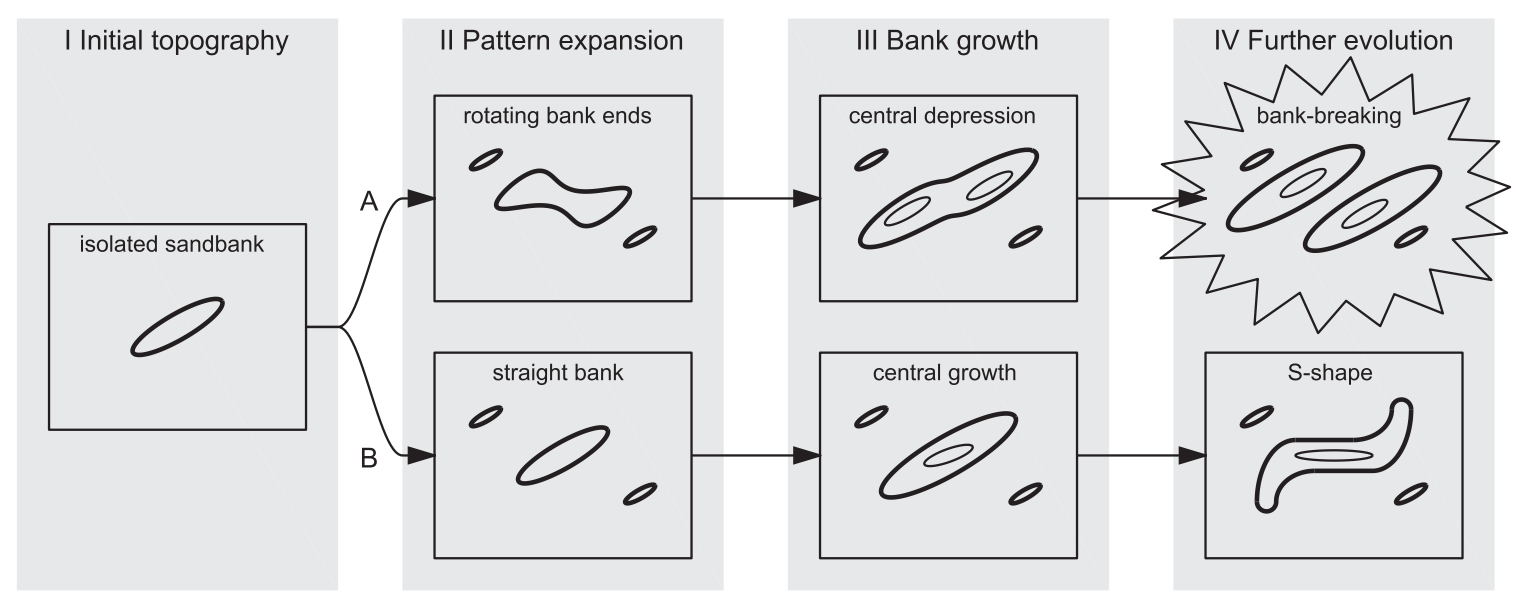

Fig. 8. Classification scheme containing two paths of bank evolution (A and B), which, depending on initial orientation and bank length, result in either bankbreaking or an S-shaped bank.

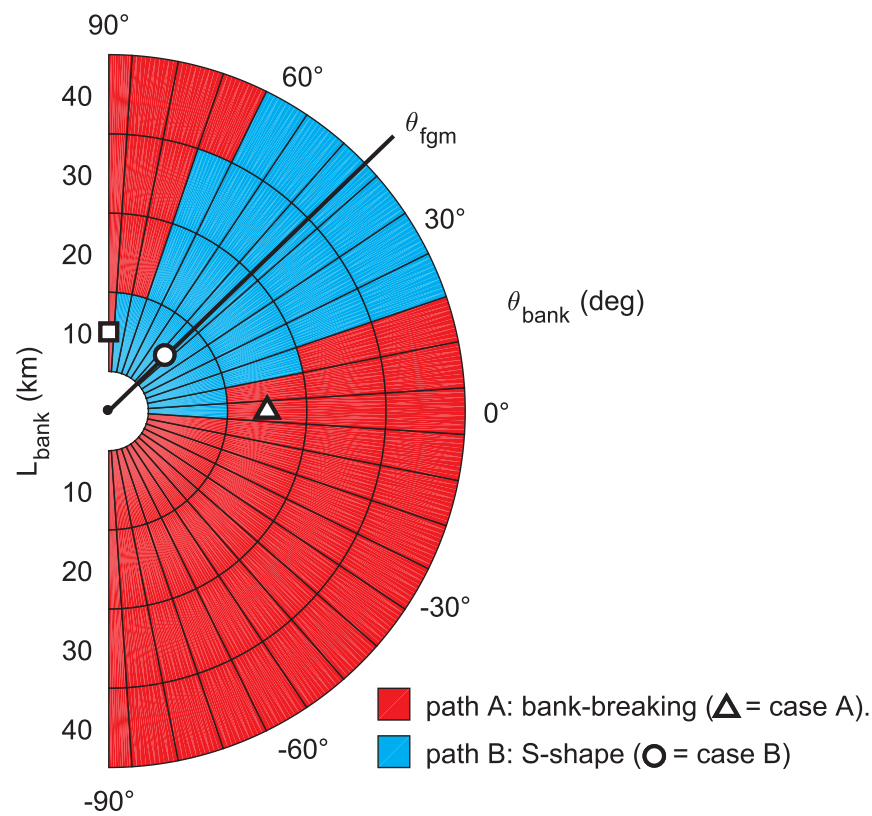

Fig. 9. Regime diagram showing bank evolution (path A or B, see Fig. 8) as a function of bank orientation $\theta_{\text {bank }}$ and length $L_{b a n k}$. Symbols are used to denote case A (triangle), case B (circle), and the case that resembles breaking according to Smith (square; to be discussed in Section 5.2). Parameter values in Tables 1 , 2.

individual growth, which is a requirement for bank breaking and thus path A. The longer the bank, the more parts into which it breaks.

Next, the bank height $h_{\text {bank }}^{*}$ controls the extent to which pattern expansion occurs in stage II. The lower the initial bank, the stronger the pattern expansion, i.e. the larger the number of banks and troughs evolving parallel to it. Furthermore, for a negative value $\left(h_{\text {bank }}^{*}=-4 \mathrm{~m}\right)$, which effectively turns the bank into a large-scale pit, pattern expansion is strongest. Finally, the bank width $B_{\text {bank }}^{*}$ controls the rate at which initial evolution takes place. Fastest evolution occurs for bank widths close to the wavelength of the fastest growing mode from linear analysis. Narrower or wider banks evolve more slowly.

\subsection{Other sensitivities}

Finally, we also investigated the sensitivity of the results to changes in various other model parameters.
- Varying the amplitude $U^{*}$ of the $M_{2}$-tidal flow mainly affects the morphological time scale; see Eq. (7). Higher flow velocities result in faster evolution.

- Increasing the wave stirring parameter $U_{w}^{*}$ enhances a diffusive mechanism, flattening the bank slopes, increasing bank width, and suppressing growth.

- Exclusion of the Coriolis effect, i.e. $f^{*}=0$, removes the preference for clockwise bank orientations relative to counterclockwise orientations. In a linear stability analysis, bank angles $\theta_{\text {bank }}$ and $-\theta_{\text {bank }}$ display identical growth rates, implying a clockwise and a counterclockwise fastest growing mode. For case A, this means that the bank ends elongate in two directions, which results in a patchy bank pattern. Case B is less affected, as it is already oriented in one of the two preferred angles.

- Increasing the bottom drag coefficient $c_{d}$, which affects the linear friction coefficient $r$, strengthens amplitude growth and accelerates the morphodynamics (breaking or evolution toward an S-shape).

- Changes in the sediment transport coefficient $\alpha^{*}$ (which incorporates grain size and sediment density) and bed porosity $\epsilon_{p o r}$ only affect the morphodynamic time scale $T_{m o r}^{*}$, which is proportional to $\left(1-\epsilon_{\text {por }}\right)$ and inversely proportional to $\alpha^{*}$; see Eq. (7).

\section{Discussion}

\subsection{Linear versus nonlinear dynamics}

Sandbank dynamics can be viewed as the superposition of linear evolution and a nonlinear correction. The former is linear in the bed amplitude (and hence in our expansion parameter $\epsilon$ ); the latter consists of all higher order contributions. To assess the importance of nonlinear sandbank dynamics, we now compare our fully nonlinear results with the linearised solution, which effectively superimposes the exponential growth or decay of the individual Fourier modes that make up the initial topography; see Eq. (27) in Section 3.6.

The bottom rows of Figs. 6 and 7 show the linear solution for cases A and $\mathrm{B}$, respectively. For both cases, the linear solution shows an expanding pattern eventually dominated by the wavelength and orientation of the fastest growing mode (see Table 2 and Roos et al. (2008)). In the nonlinear results (top rows of Figs. 6 and 7), this pattern expansion is weaker and also bank growth is suppressed. Furthermore, bank breaking in case A occurs in both the nonlinear and linear solutions. Alternatively, the S-shape emerging in case B is clearly a nonlinear effect, i.e. involving interaction among the Fourier modes. This may be a manifestation of an along-crest instability also found by Yuan et al. (2017).

We conclude that rotation of bank ends and pattern expansion can 

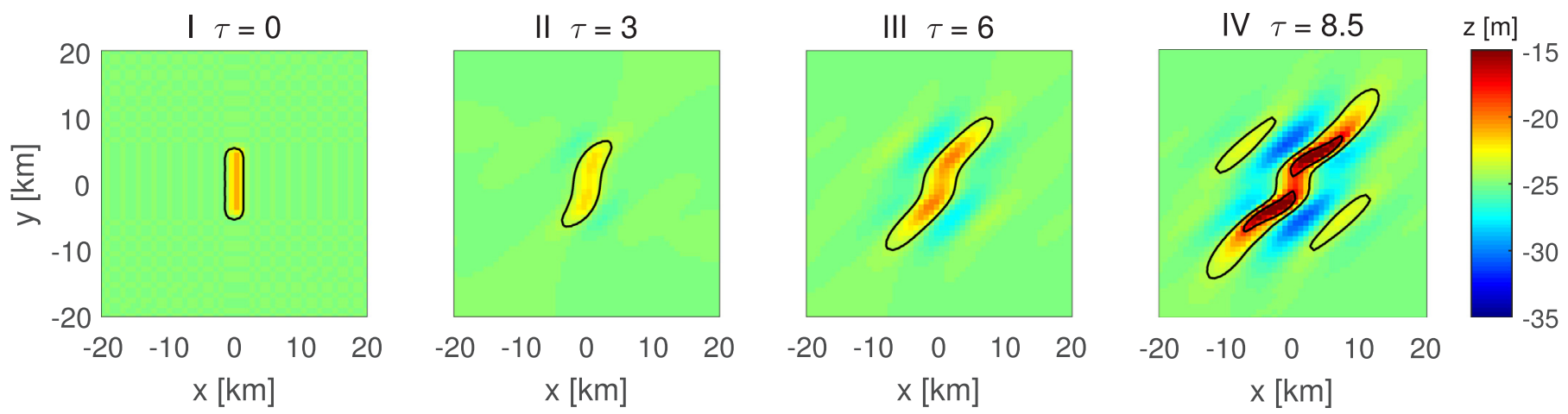

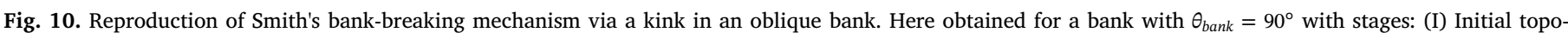

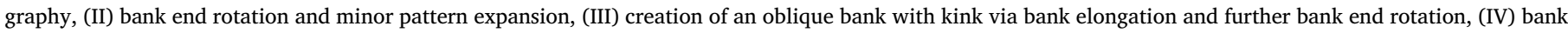
breaking with the kink turning into a widening depression, separating the two former bank ends. Black lines indicate the $18 \mathrm{~m}$ and $24 \mathrm{~m}$ depth contours.

also be captured in a linear model, but the suppression of pattern expansion and bank growth as well as the evolution into an S-shape clearly require the inclusion of nonlinear dynamics.

\subsection{Comparison with bank-break hypotheses from literature}

Both Caston (1972; see Fig. 1) and case A describe breaking of a bank parallel to the tidal flow. It should be noted that breaking in three rather than two parts required extending the bank length of case A, from $20 \mathrm{~km}$ to $40 \mathrm{~km}$. Furthermore, Caston's intermediate stages do not necessarily match ours. In fact, some of these stages appear to show up in our path B, which leads to an S-shape rather than to breaking.

The breaking mechanism of an anticlockwise oriented bank with kink, as described by Smith (1988; see Fig. 2) has been reproduced in our model. In our results, the kink arose from rotation of a bank initially oriented perpendicular to the tidal flow (Fig. 10). Interestingly, anticlockwise oriented banks without an initial kink did not break in our model (case B). This suggests that breaking requires either an initial disturbance or an unnatural orientation.

Importantly, our model has reproduced bank-breaking without inclusion of sandwave dynamics. Incorporating sandwaves, playing an important role in the bank-break mechanism by Smith (1988), would require a three-dimensional flow model (Hulscher, 1996). Nevertheless, we have shown that sandwave dynamics is no prerequisite for bankbreaking, although they could modify the mechanism.

The dynamics of non-breaking banks resemble those produced in earlier long-term modelling studies. Under simplified conditions, Huthnance (1982b) found elongation and an S-shape for initially oblique banks, and separate evolution of crests for perpendicular banks. Furthermore, Yuan et al. (2017) showed that initially straight banks with a fixed wave length develop along-crest instabilities. The results of both studies fit in our classification scheme (Fig. 8).

\subsection{Comparison with North Sea banks}

In the previous section, we have shown that our model is capable of reproducing bank-breaking mechanisms that were based on observations of the Norfolk (Caston, 1972) and Hinder banks (Smith, 1988) in the North Sea. Besides, the along-bank irregularities showing up in our model results are also observed at the Norfolk Banks (Fig. 11). Specifically, the Ower Bank and Indefatigable Banks display a kink in their topography, which we found after rotation of the bank ends in stage II (Fig. 10). Furthermore, the Swarte Bank and the Indefatigable Banks show separately growing features, corresponding to stage III of path A (breaking path). Finally, the meandering shapes of both the Leman Bank and Ower Bank resembles path B, evolving into an S-shape.

Finally, it should be noted that the bathymetric chart in Fig. 11 provides only a snapshot of an evolution that takes place on the time

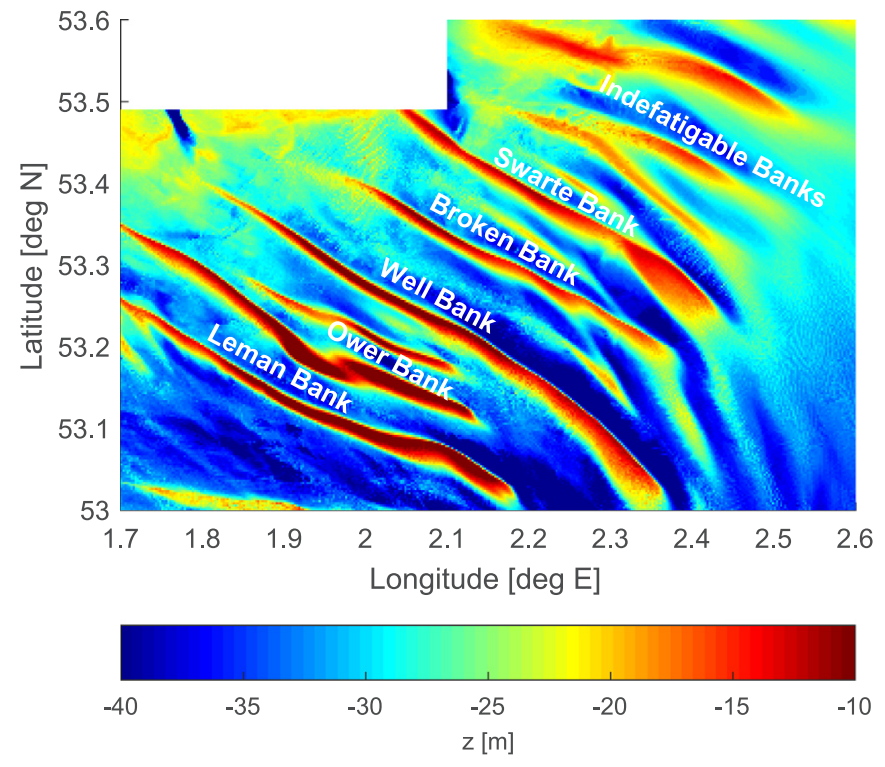

Fig. 11. Map of the bathymetry of the Norfolk banks, UK Continental Shelf. Source: survey from 1991 by UKHO (see Acknowledgement).

scale of centuries and longer. Along with uncertainties in hydrodynamic conditions over these time scales and other complications (shelf slope, coastline, other banks), this precludes a more detailed comparison.

\subsection{Model assumptions and properties}

Domain size An important property of our model is the spatially periodic domain, implying that the sandbank recurs over a distance $L_{\text {dom }}^{*}$ in the $x^{*}$ and $y^{*}$-directions. Immediately associated with this is the lowest wave number $k_{\min }^{*}=2 \pi / L_{d o m}^{*}$ (here expressed in dimensional form) in our discrete Fourier representation; see Eq. (20). In fact, domain size acts as a numerical parameter that should be chosen sufficiently large to avoid unwanted hydro- and morphodynamic interaction between adjacent banks. We have achieved this by taking $L_{d o m}^{*}=100 \mathrm{~km}$. To support our choice: for the initial topography of case A, maximum values of the dimensionless (tide-averaged) vorticity $\langle\eta\rangle$ outside a radius of $25 \mathrm{~km}$ from the bank's centre are in the order of $10^{-4}$, which is a factor $10^{3}$ smaller than the maximum value in the domain.

Initial topography Our choice of initial topography, as depicted in Figs. 3 and 4, is inspired by observations and the literature on bankbreaking mechanisms. This explains our focus on a single bank as well as the chosen shape. Varying the four bank parameters enables us to cover a wide range of bank topographies. This includes banks that may have formed in times of different conditions or be of other origin. 


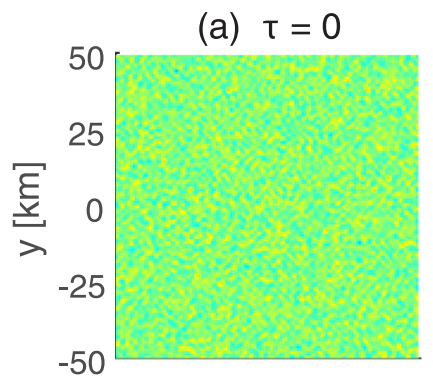

(b) $T=3$

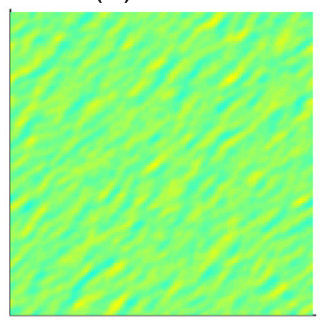

(c) $\tau=5$

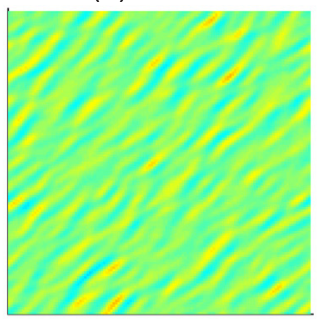

(d) $\tau=7$

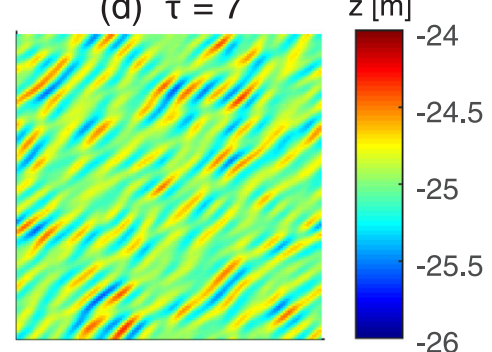

(e) $\tau=9$

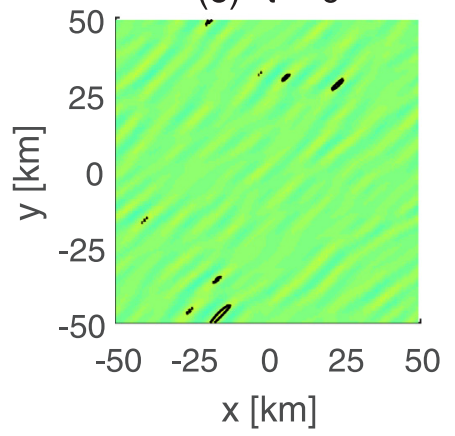

(f) $\tau=11$

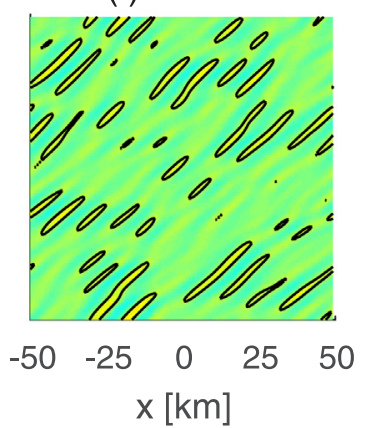

(g) $\tau=13$

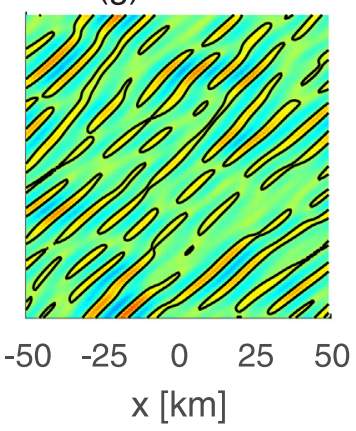

(h) $\tau=15$

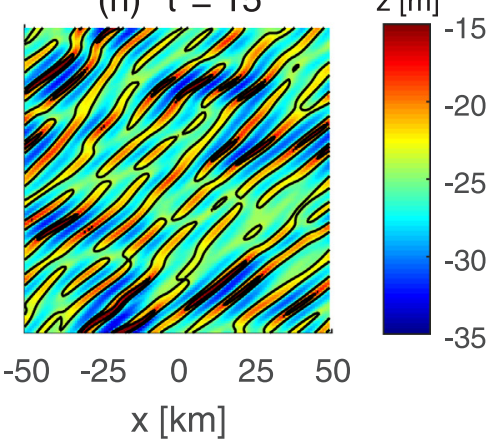

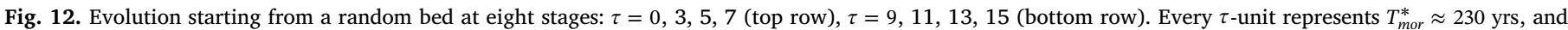
black lines indicate the $24 \mathrm{~m}$-depth contour. Unlike earlier figures, the entire model domain ( $100 \mathrm{~km})$ is plotted and each row has a different colorbar.

Furthermore, by placing our bank in an otherwise smooth domain, we exclude interaction with features already present on the surrounding bed. To explore this further, we have conducted two additional simulations.

- Starting from a flat bed with random noise only (here of amplitude $0.25 \mathrm{~m}$, i.e. $1 \%$ of the ambient water depth) shows the gradual formation of a rather regular sandbank pattern with orientation and spacing corresponding to linear theory. Irregularities result from the initial noise and nonlinear interaction, including S-shape development (Fig. 12).

- Adding such noise around the initial bank produces hardly any differences with the 'smooth' runs, as the noise evolves more slowly than the bank (simulation not shown).

Expansion parameter Apart from the relative bank amplitude $\epsilon$, used as expansion parameter in our hydrodynamic solution method, the model contains two other small parameters: the (squared) Froude number $\mathrm{Fr}^{2} \approx 10^{-3}$ and the ratio $\gamma \approx 10^{-6}$ of hydrodynamic and morphodynamic time scales (values corresponding to parameter settings in Table 2). We assume that these parameters are smaller than $\epsilon^{j}$ for any $j=1,2, \cdots, J$. For $\gamma$, this seems reasonable, but we are aware that depending on $\epsilon$ and physical setting, this may be violated by $\mathrm{Fr}^{2}$. Also, we cannot make any general statements on the convergence properties of our solution method; there is no guarantee that it converges for $\epsilon<1$.

Equilibria On time scales longer than the focus of our study, the simulations generally develop instabilities and do not converge to an equilibrium. It is unclear whether this is due to the (hydrodynamic) solution method or the choice of numerical parameters $(M, \Delta \tau)$. Thus, we cannot compare with the equilibria obtained in earlier nonlinear studies on smaller model domains, such as the static or migrating profiles with parallel depth contours by Roos et al. (2004) and the spatially meandering crests that oscillate in time found by Yuan et al. (2017).

Sea level rise By keeping the ambient water depth $H^{*}$ and the tidal/ wave conditions constant in our simulations, we ignore the effects of sea level rise and isostatic rebound taking place on the time scale of bank evolution. Including this would enable other types of dynamics, e.g. an active sandbank becoming quasi-active or inactive, as found in the idealised numerical model study by Yuan and de Swart (2017).

Sediment transport Although our sediment transport formulation in Eq. (4) is highly idealised, the major physical elements are included: bed load, faster-than-linear dependency on flow velocity, bed slope effect and depth-dependent wave stirring. Regarding schematisations, we have neglected the critical shear stress and bed slope anisotropy, which are not critical but have a quantitative effect on the linear growth properties (Yuan et al., 2016). Analogous to Roos et al. (2004), wave stirring has been included using a stirring term $\frac{1}{2} U_{w}^{* 2}\left[h^{*} / H^{*}\right]^{-2}$ in the transport formula (4). This stirring term is proportional to the square of a velocity $U_{w}^{*}\left[h^{*} / H^{*}\right]^{-1}$, which is inversely proportional to water depth (i.e., power -1 , close to the power -0.8 derived by Calvete et al., 2002). A more detailed approach would be to combine linear wave theory with a wave energy balance including dissipation and wind forcing terms (e.g., Vis-Star et al., 2007). The settling lag of suspended sediment is likely to lower and smooth bank profiles (Roos et al., 2004). Finally, we expect that incorporating non-uniform sediment, apart from providing information about the spatial grain size distribution, will not affect bank shape evolution (Walgreen et al., 2004; Roos et al., 2007).

\section{Conclusions}

We have developed an idealised process-based morphodynamic model for the transient evolution of tidal sandbanks. Specifically, it includes tide-topography interactions (through bottom friction and the Coriolis effect), captures long-term nonlinear dynamics and allows for topographies varying in both horizontal directions. As a novelty, the hydrodynamic solution is obtained from a truncated expansion in the ratio of bank elevation (w.r.t. to mean depth) and mean water depth.

Based on our model results, we observe that banks either break or attain an S-shape (Fig. 8). Both types follow a specific four-stage evolution, denoted by path A and B. Path A: Starting from an initially straight bank, (II) bank ends rotate toward the preferred bank orientation from linear analysis and (III) grow separately around a 
depression at the central part, which leads to (IV) bank-breaking. Path B: (I) An initial straight bank (II) retains its shape. (III) The central grows fastest and aligns with the principal direction of the tidal flow, which leads to (IV) an S-shaped bank.

Whether bank-breaking (path A) occurs, depends on the initial bank orientation and length. Specifically, two conditions must be met. First, the initial bank orientation should differ from the preferred bank orientation from linear analysis. Otherwise, the bank ends will not rotate. Second, the bank should be sufficiently long for the elevated crests to grow separately. If these conditions are not satisfied, the bank will evolve toward an S-shape (path B). These conditions can be visualised in a so-called regime diagram (Fig. 9).

In addition to these path-dependent dynamics, the banks also display pattern expansion and elongation. Details depend on initial topography. Comparison between linear and nonlinear dynamics has shown that nonlinear dynamics are essential to damp bank growth and to capture the evolution toward an S-shape.

The here presented breaking mechanism (path A) is consistent with the observation-based breaking mechanism by Smith (1988). Furthermore, we were able to reproduce bank-breaking under the conditions in Caston (1972), although his stages do not match ours. Alternatively, the evolution toward an S-shape (path B) is consistent with model results by Huthnance (1982b). Finally, the breaking and S-shapes in our model results resemble the plan view characteristics of, e.g., the Norfolk Banks in the North Sea.

\section{Acknowledgements}

This work contains public sector information, licensed under the Open Government Licence v2.0, from the UKHO (Fig. 11).

This work is part of the research programme SMARTSEA with project number 13275, which is (partly) financed by the Netherlands Organisation for Scientific Research (NWO).

We thank Huib de Swart and two anonymous Reviewers for their comments.

\section{Appendix A. Details of the initial bank topography}

The initial bank topography, as introduced in Section 2.5 and shown in Figs. 3 and 4, is given by

$z^{*}=-H^{*}+h_{\text {bank }}^{*} \chi\left(x^{*}, y^{*}\right)$,

with dimensionless shape function $\chi$. This function specifies both the cross-sectional Gaussian shape in the central part and the two-dimensional Gaussian shape at the bank ends:

$\chi= \begin{cases}\exp \left(-\pi \widetilde{y}^{* 2} / B_{b a n k}^{* 2}\right) & \text { if } \mid \widetilde{x}^{* 1} \leq X^{*}, \\ \exp \left(-\pi\left[\left(\left|\widetilde{x}^{*}\right|-X^{*}\right)^{2}+\widetilde{y}^{* 2}\right] / B_{b a n k}^{* 2}\right) & \text { if } \mid \widetilde{x}^{* 1}>X^{*} .\end{cases}$

Here, $X^{*}=\frac{1}{2}\left(L_{b a n k}^{*}-B_{b a n k}^{*}\right)$ is half the length of the bank's central part and we have introduced the (rotated) along-bank and cross-bank coordinates $\left(\widetilde{x}^{*}, \widetilde{y}^{*}\right)$ according to

$\left[\begin{array}{c}\tilde{x}^{*} \\ \tilde{y}^{*}\end{array}\right]=\left[\begin{array}{cc}\cos \theta_{\text {bank }} & \sin \theta_{\text {bank }} \\ -\sin \theta_{\text {bank }} & \cos \theta_{\text {bank }}\end{array}\right]\left[\begin{array}{c}x^{*} \\ y^{*}\end{array}\right]$

\section{Appendix B. First order flow solution}

The first order solution $\phi_{1}$ describes the linear flow response to the bed perturbation $h_{1}$. Evaluating the vorticity Eq. (22) at $\mathcal{O}(\epsilon)$ shows that the linear problem (24) for $\eta_{1}$ takes the following form:

$\mathscr{L}_{0} \eta_{1}=\underbrace{\left[\frac{f}{h_{0}} \frac{\partial h_{1}}{\partial x}-\frac{r}{h_{0}^{2}} \frac{\partial h_{1}}{\partial y}\right]}_{R_{1}} u_{0}+\underbrace{\left[\frac{f}{h_{0}} \frac{\partial h_{1}}{\partial y}+\frac{r}{h_{0}^{2}} \frac{\partial h_{1}}{\partial x}\right]}_{S_{1}} v_{0}$,

with linear operator $\mathscr{L}_{0}=\frac{\partial}{\partial t}+u_{0} \frac{\partial}{\partial x}+v_{0} \frac{\partial}{\partial y}+r / h_{0}$ as already introduced in Section 3.4. Note that the terms $R_{0} u_{1}$ and $S_{0} v_{1}$ vanish, because the lowest order topography is spatially uniform.

To account for the spatiotemporal variations in $\eta_{1}$, we write

$\eta_{1}(x, y, t)=\sum_{m=-M}^{M} \sum_{n=-M}^{M} \hat{\eta}_{1 m n}(t) \exp \left(i\left[k_{m} x+l_{n} y\right]\right)$,

in which the spatial Fourier coefficients $\hat{\eta}_{1 m n}$ are then further expanded in a time series according to

$\hat{\eta}_{1 m n}(t)=\sum_{p=-P}^{P} E_{1 m n p} \exp (i p t)$.

Herein, $E_{1 m n p}$ are the (complex) spatiotemporal Fourier components and $P$ is the temporal truncation number.

Because the right-hand side of Eq. (B.1) is linear in $h_{1}$, the spatial modes (denoted by subscripts $m$ and $n$ ) do not interact order of approximation. Hence, the Fourier components of the vorticity of each spatial mode can be obtained separately from a set of linear equations:

$L_{m n} \vec{E}_{1 m n}=\vec{b}_{1 m n}$.

Here, we have introduced the $(2 P+1) \times(2 P+1)$ matrix $L_{m n}$, which for $P=2$ is given by 
$L_{m n}=\left[\begin{array}{ccccc}\delta_{-2}+A_{0} & A_{-1} & A_{-2} & 0 & 0 \\ A_{1} & \delta_{-1}+A_{0} & A_{-1} & A_{-2} & 0 \\ A_{2} & A_{1} & \delta_{0}+A_{0} & A_{-1} & A_{-2} \\ 0 & A_{2} & A_{1} & \delta_{1}+A_{0} & A_{-1} \\ 0 & 0 & A_{2} & A_{1} & \delta_{2}+A_{0}\end{array}\right]$,

with shorthand notation $A_{p}=i k_{m} U_{0 p}+i l_{n} V_{0 p}$ and $\delta_{p}=i p+r / h_{0}$ for $p=-P, \cdots, P$. Furthermore, in Eq. (B.4), $\vec{E}_{1 m n}$ is a column vector with elements $E_{1 m n p}$, and so is the forcing term $\vec{b}_{1 m n}$ with elements

$b_{1 m n p}=\left[\frac{f i k_{m}}{h_{0}}-\frac{r i l_{n}}{h_{0}^{2}}\right] U_{0 p}+\left[\frac{f i l_{n}}{h_{0}}+\frac{r i k_{m}}{h_{0}^{2}}\right] V_{0 p}$.

To translate the thus obtained vorticity $\hat{\eta}_{1 m n}$ back to tidal flow components, we combine the continuity Eq. (11) with the definition of vorticity in Eq. (21). This leads to

$\hat{u}_{1 m n}=\frac{i l_{n} \hat{\eta}_{1 m n}+i k_{m} \hat{a}_{1 m n}}{k_{m}^{2}+l_{n}^{2}}, \hat{v}_{1 m n}=\frac{-i k_{m} \hat{\eta}_{1 m n}+i l_{n} \hat{a}_{1 m n}}{k_{m}^{2}+l_{n}^{2}}$,

with $\hat{a}_{1 m n}=\left(i k_{m} u_{0}+i l_{n} v_{0}\right) \hat{h}_{1 m n} / h_{0}$.

\section{Appendix C. Higher order flow solutions}

The higher order flow solutions $\phi_{j}$ with $j=2,3, \cdots, J$ describe subsequent nonlinear contribution terms to the hydrodynamic solution. Evaluating the vorticity Eq. (22) at $\mathcal{O}\left(\epsilon^{j}\right)$ shows that the forcing terms in the linear problem (24) for $\eta_{j}$ contain convolution sums of lower order quantities:

$\mathscr{L}_{0} \eta_{j}=\sum_{j^{\prime}=1}^{j}(\underbrace{R_{j^{\prime}} u_{j-j^{\prime}}+S_{j^{\prime}} v_{j-j^{\prime}}}_{\begin{array}{c}\text { Coriolis and } \\ \text { frictional torques }\end{array}}-\underbrace{\left[u_{j^{\prime}} \frac{\partial \eta_{j-j^{\prime}}}{\partial x}+v_{j^{\prime}} \frac{\partial \eta_{j-j^{\prime}}}{\partial y}\right]}_{\text {advection }}-\underbrace{\mu_{j^{\prime}} \eta_{j-j^{\prime}}}_{\begin{array}{c}\text { depth effect } \\ \text { on friction }\end{array}})$.

Herein, $R_{j^{\prime}}, S_{j^{\prime}}$ and $\mu_{j^{\prime}}$ are the $j^{\prime}$-th order expansions of the functions $R, S$ and $\mu$, given by

$R_{j}=\left(\frac{-h_{1}}{h_{0}}\right)^{j-1}\left[\frac{f}{h_{0}} \frac{\partial h_{1}}{\partial x}-\frac{j r}{h_{0}^{2}} \frac{\partial h_{1}}{\partial y}\right], S_{j}=\left(\frac{-h_{1}}{h_{0}}\right)^{j-1}\left[\frac{f}{h_{0}} \frac{\partial h_{1}}{\partial y}+\frac{j r}{h_{0}^{2}} \frac{\partial h_{1}}{\partial x}\right], \mu_{j}=\left(\frac{-h_{1}}{h_{0}}\right)^{j} \frac{r}{h_{0}}$.

Contrary to the first order flow solution (Appendix B), the spatial modes in the nonlinear forcing terms in Eq. (C.1) do interact. Hence, spatial convolution sums need to be evaluated in all forcing terms, for which we use the pseudospectral method (Boyd, 2000). Furthermore, an additional temporal convolution sum needs to be evaluated in the advective term.

Once all forcing terms on the right-hand side of Eq. (C.1) are known, the $j$-th order vorticity $\eta_{j}$ and flow $\left(u_{j}\right.$, $v_{j}$ ) is computed in a way similar to the first order hydrodynamic solution, see Eqs. (B.4)-(B.7). This results in the Fourier coefficients for the tidal flow (except for the spatially uniform part):

$\hat{u}_{j m n}=\frac{i l_{n} \hat{\eta}_{j m n}+i k_{m} \hat{a}_{j m n}}{k_{m}^{2}+l_{n}^{2}}, \hat{v}_{j m n}=\frac{-i k_{m} \hat{\eta}_{j m n}+i l_{n} \hat{a}_{j m n}}{k_{m}^{2}+l_{n}^{2}}$,

with $\hat{a}_{j m n}=\left(i k_{m}\left\{h_{1} u_{j-1}\right\}_{m n}+i l_{n}\left\{h_{1} v_{j-1}\right\}_{m n}\right) / h_{0}$. Here, $\left\{h_{1} u_{j-1}\right\}_{m n}$ and $\left\{h_{1} v_{j-1}\right\}_{m n}$ refer to the contributions of the spatial convolution of the quantities $h_{1} u_{j-1}$ and $h_{1} v_{j-1}$ to mode $(m, n)$.

The spatially uniform part of the flow must be treated separately. To this end, we apply the spatially averaged momentum equations to obtain:

$\left[\begin{array}{cc}i p+\mu_{0} & -f \\ f & i p+\mu_{0}\end{array}\right]\left[\begin{array}{l}U_{j 00 p} \\ V_{j 00 p}\end{array}\right]=\left[\begin{array}{l}-C_{j 00 p} \\ -D_{j 00 p}\end{array}\right]$

in which $\mu_{0}=r / h_{0}$ according to Eq. (C.2), whereas $C_{j 00 p}$ and $D_{j 00 p}$ are the $p$-th order temporal Fourier coefficients of the following spatially uniform yet time-dependent quantities:

$\hat{c}_{j 00}=\sum_{j^{\prime}=1}^{j}\left\{\mu_{j^{\prime}} u_{j-j^{\prime}}+u_{j^{\prime}} \frac{\partial u_{j-j^{\prime}}}{\partial x}+v_{j^{\prime}} \frac{\partial u_{j-j^{\prime}}}{\partial y}\right\}_{00}, \hat{d}_{j 00}=\sum_{j^{\prime}=1}^{j}\left\{\mu_{j^{\prime}} v_{j-j^{\prime}}+u_{j^{\prime}} \frac{\partial v_{j-j^{\prime}}}{\partial x}+v_{j^{\prime}} \frac{\partial v_{j-j^{\prime}}}{\partial y}\right\}_{00}$.

The solution to the linear system in Eq. (C.4) is given by

$U_{j 00 p}=\frac{\left(i p+\mu_{0}\right) C_{j 00 p}+f D_{j 00 p}}{\left(i p+\mu_{0}\right)^{2}+f^{2}}, V_{j 00 p}=\frac{-f C_{j 00 p}+\left(i p+\mu_{0}\right) D_{j 00 p}}{\left(i p+\mu_{0}\right)^{2}+f^{2}}$.

\section{References}

Atalah, J., Fitch, J., Coughlan, J., Chopelet, J., Coscia, I., Farrell, E., 2013. Diversity of demersal and megafaunal assemblages inhabiting sandbanks of the Irish Sea. Mar. Biodiv. 43, 121-132.

Besio, G., Blondeaux, P., Vittori, G., 2006. On the formation of sand waves and sand banks. J. Fluid Mech. 557, 1-27.
Boyd, J.P., 2000. Chebyshev and Fourier Spectral Methods, 2nd eda. Dover Publications, New York.

Brière, C., Roos, P.C., Garel, E., Hulscher, S.J.M.H., 2010. Modelling the morphodynamics of the Kwinte Bank, subject to sand extraction. J. Coast. Res. 51, 117-126.

Calvete, D., de Swart, H.E., Falqués, A., 2002. Effect of depth-dependent wave stirring on the final amplitude of shoreface-connected sand ridges. Cont. Shelf Res. 22, 2763-2776.

Caston, V.N.D., 1972. Linear sand banks in the Southern North Sea. Sedimentology 18, 
$63-78$.

Caston, V.N.D., Stride, A.H., 1970. Tidal sand movement between some linear sand banks in the North Sea off northeast Norfolk. Mar. Geol. 9, M38-M42.

De Vriend, H.J., 1990. Morphological processes in shallow tidal seas. In: Cheng, R.T (Ed.), Residual Currents and Long-term Transport Number 38 in Coastal and Estuarine Studies. Springer, New York, pp. 276-301.

Deleu, S., van Lancker, V.R.M., van den Eynde, D., Moerkerke, G., 2004. Morphodynamic evolution of the kink of an offshore tidal sandbank: the Westhinder Bank (Southern North Sea). Cont. Shelf Res. 24, 1587-1610.

Dolphin, T.J., Vincent, C.E., Coughlan, C., Rees, J.M., 2007. Variability in sandbank behaviour at decadal and annual time-scales and implications for adjacent beaches. J. Coast. Res. 50, 731-737.

Dyer, K.R., Huntley, D.A., 1999. The origin, classification and modelling of sand banks and ridges. Cont. Shelf Res. 19, 1285-1330.

Fairley, I., Masters, I., Karunarathna, H., 2016. Numerical modelling of storm and surge events on offshore sandbanks. Mar. Geol. 371, 106-119.

Horrillo-Caraballo, J.M., Reeve, D.E., 2008. Morphodynamic behaviour of a nearshore sandbank system:the Great Yarmouth Sandbanks. U. K. Mar. Geol. 254, 91-106.

Hulscher, S.J.M.H., 1996. Tidal-induced large-scale regular bed form patterns in a threedimensional shallow water model. J. Geophys. Res. 101, 20727-20744.

Hulscher, S.J.M.H., de Swart, H.E., de Vriend, H.J., 1993. The generation of offshore tidal sand banks and sand waves. Cont. Shelf Res. 13, 1183-1204.

Huthnance, J.M., 1973. Tidal current asymmetries over the Norfolk Sandbanks. Estuar. Coast. Mar. Sci. 1, 89-99.

Huthnance, J.M., 1982a. On one mechanism forming linear sand banks. Estuar. Coast. Shelf Sci. 14, 79-99.

Huthnance, J.M., 1982b. On the formation of sand banks of finite extent. Estuar. Coast. Shelf Sci. 15, 277-299.

Kaiser, M.J., Bergmann, M., Hinz, H., Galanidi, M., Shucksmith, R., Rees, E.I.S., Darbyshire, T., Ramsay, K., 2004. Demersal fish and epifauna associated with sandbank habitats. Est. Coast. Shelf Sci. 60, 445-456.

Kenyon, N.H., Belderson, R.H., Stride, A.H., Johnson, M.A., 1981. Offshore tidal sandbanks as indicators of net sand transport and as potential deposits. In: Nio, S.-D. Schüttenhelm, R.T.E., van Weering, T.C.E. (Eds.), Holocene Marine Sedimentation in the North Sea Basin. Blackwell Publishing Ltd, pp. 257-268.

van Leeuwen, S., Dodd, N., Calvete, D., Falqués, A., 2007. Linear evolution of a shoreface nourishment. Coast. Eng. 54, 417-431.

Pan, S., MacDonald, N., Williams, J., O'Connor, B.A., Nicholson, J., Davies, A.M., 2007. Modelling the hydrodynamics of offshore sandbanks. Cont. Shelf Res. 27, 1264-1286.

Pattiaratchi, C., Collins, M., 1987. Mechanisms for linear sandbank formation and maintenance in relation to dynamical oceanographic observations. Prog. Oceanogr. $19,117-176$.

Robinson, I.S., 1981. Tidal vorticity and residual circulation. Deep Sea Res. 28, 195-212.

Roos, P.C., Blondeaux, P., Hulscher, S.J.M.H., Vittori, G., 2005. Linear evolution of sandwave packets. J. Geophys. Res. 110 (F04S14).

Roos, P.C., Hulscher, S.J.M.H., 2003. Large-scale seabed dynamics in offshore morphology: modeling human intervention. Rev. Geophys. 41, 1010.
Roos, P.C., Hulscher, S.J.M.H., Knaapen, M.A.F., van Damme, R.M.J., 2004. The crosssectional shape of tidal sandbanks:modeling and observations. J. Geophys. Res. 109 (F02003).

Roos, P.C., Hulscher, S.J.M.H., de Vriend, H.J., 2008. Modelling the morphodynamic impact of offshore sandpit geometries. Coast. Eng. 55, 704-715.

Roos, P.C., Wemmenhove, R., Hulscher, S.J.M.H., Hoeijmakers, H.W.M., Kruyt, N.P., 2007. Modeling the effect of nonuniform sediment on the dynamics of offshore tidal sandbanks. J. Geophys. Res. 112 (F02011).

Sanay, R., Voulgaris, G., Warner, J.C., 2007. Tidal asymmetry and residual circulation over linear sandbanks and their implication on sediment transport: a process-oriented numerical study. J. Geophys. Res. 112, C12015.

Sekine, M., Parker, G., 1992. Bed-load transport on transverse slope. I. J. Hydraul. Eng. $118,513-535$.

Smith, D.B., 1988. Stability of an offset kink in the North Hinder Bank. In: De Boer, P., Van Gelder, A., Nio, S.-D. (Eds.), Tide-Influenced Sedimentary Environments and Facies. D. Reidel Publishing Company, Dordrecht, pp. 65-78.

Tambroni, N., Blondeaux, P., 2008. Sand banks of finite amplitude. J. Geophys. Res. 113, C10028.

Van den Eynde, D., Giardino, A., Portilla, J., Fettweis, M., Francken, F., Monbaliu, J., 2010. Modelling the effects of sand extraction, on sediment transport due to tides, on the Kwinte Bank. J. Coast. Res. 51, 101-116.

Van Lancker, V.R.M., Bonne, W.M.I., Velegrakis, A.F., Collins, M.B., 2010. Aggregate extraction from tidal sandbanks: is dredging with nature an option? J. Coast. Res. 51, $53-61$.

Van Rijn, L.C., 1993. Principles of Sediment Transport in Rivers, Estuaries and Coastal Seas. Aqua Publications, Amsterdam.

Vis-Star, N.C., de Swart, H.E., Calvete, D., 2007. Effect of wave-topography interactions on the formation of sand ridges on the shelf. J. Geophys. Res. 112, C06012.

Walgreen, M., Swart, H.E.D., Calvete, D., 2004. A model for grain-size sorting over tida sand ridges. Ocean Dyn. 54, 374-384.

Whitehouse, R.J.S., Harris, J.M., Sutherland, J., Rees, J., 2011. The nature of scour development and scour protection at offshore windfarm foundations. Mar. Pollut. Bull. $62,73-88$.

Williams, J.J., MacDonald, N.J., O'Connor, B.A., Pan, S., 2000. Offshore sand bank dynamics. J. Mar. Syst. 24, 153-173.

Yuan, B., de Swart, H.E., 2017. Effect of sea level rise and tidal current variation on the long-term evolution of offshore tidal sand ridges. Mar. Geol. 390, 199-213.

Yuan, B., de Swart, H.E., Panadès, C., 2016. Sensitivity of growth characteristics of tida sand ridges and long bed waves to formulations of bed shear stress, sand transport and tidal forcing: a numerical model study. Cont. Shelf Res. 127, 28-42.

Yuan, B., de Swart, H.E., Panadès, C., 2017. Modeling the finite-height behavior of offshore tidal sand ridges, a sensitivity study. Cont. Shelf Res. 137, 72-83.

Zimmerman, J.T.F., 1981. Dynamics, diffusion and geomorphological significance of tidal residual eddies. Nature 290, 549-555.

Zimmerman, J.T.F., 1982. On the Lorentz linearization of a quadratically damped forced oscillator. Phys. Lett. A 89, 123-124. 